\title{
Breaking the Barrier to Slow Water Exchange Rates for Optimal Magnetic Resonance Detection of paraCEST Agents
}

W. Shirangi Fernando ${ }^{\dagger}$, André F. Martins ${ }^{\dagger}$, Piyu Zhao ${ }^{\dagger}$, Yunkou Wu ${ }^{\ddagger}$, Garry E. Kiefer ${ }^{\dagger, \S}$, Carlos Platas-Iglesias $\xi$ and A. Dean Sherry ${ }^{\ddagger *}$

${ }^{\dagger}$ Department of Chemistry, 8oo West Campbell Road, Richardson, Texas 75080, United States

${ }^{\ddagger}$ Advanced Imaging Research Center, University of Texas Southwestern Medical Center, Dallas, Texas 75390, United States

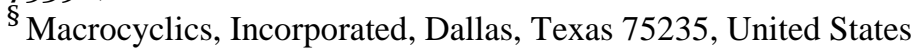

${ }^{\xi}$ Grupo QUICOOR, Centro de Investigaciones Científicas Avanzadas (CICA) and Departamento de Química Fundamental, Universidade da Coruña, Campus da Zapateira, Rúa da Fraga 10, 15008 A Coruña, Spain

\section{SUPPORTING INFORMATION}

\section{Contents}

Methods

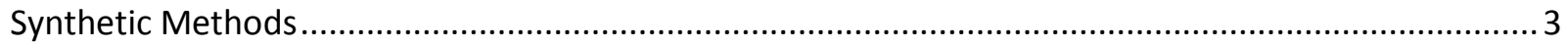

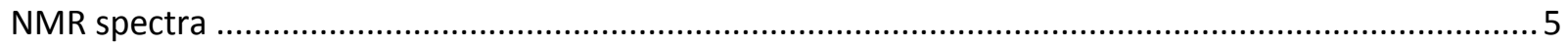

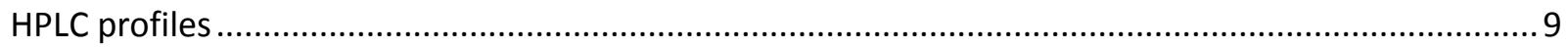

CEST spectra, Structure elucidation, Computed POTENtials and in vivo supporting information ..........11

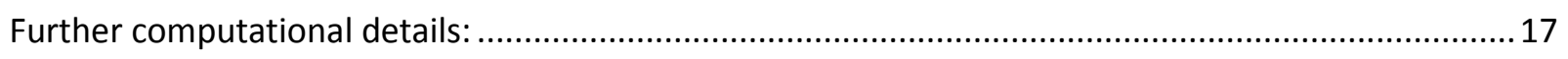

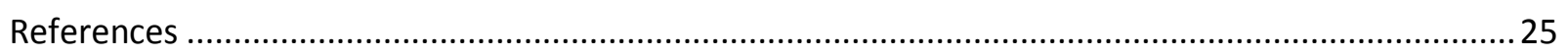

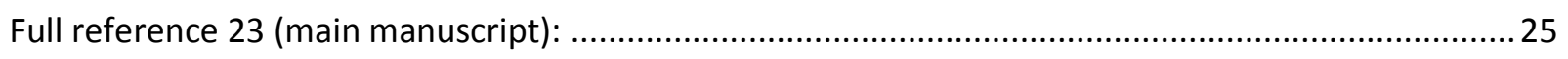




\section{METHODS}

General Remarks. All reagents and solvents were purchased from commercial sources and used as received unless otherwise stated. Deionized water $(18 \mathrm{M} \Omega \cdot \mathrm{cm})$ purified by an ultra-pure water system (Milli-Q, Millipore) was used in all experiments. All NMR spectra and CEST spectra were recorded on Bruker Avance III 400 NMR system equipped with a 5 $\mathrm{mm}$ broadband probe. Melting points were recorded on a Fisher/Johns melting point apparatus without correction. A Fischer Science Education $\mathrm{pH}$ meter equipped with a glass electrode was used to measure the solution $\mathrm{pH}$ values. Luminescence measurements were done using a F920 Edinburgh fluorescence spectrometer. Analytical HPLC was performed on an Agilent 1220 system using a Restek Ultra C18 reversed phase column (4.6×100 mm). Preparative HPLC was performed on a Waters DeltaPrep preparative chromatography system using a luna $5 \mu \mathrm{C} 18$ reversed phase column.

Synthetic details. The ligands were prepared by alkylation of cyclen by a benzylprotected glutamic acid followed by addition of amino methyl phosphonate groups via a coupling reaction. Removal of the benzyl groups yielded ligand L1 (see scheme 1) in good yield. By controlled addition of lithium hydroxide to ligand Li and analytical HPLC monitoring, the monoester version, L2, was also obtained in good yield. The lanthanide complexes EuL1 and EuL2 were prepared by standard complexation procedures in water and were purified by preparative HPLC. All intermediates and final complexes were characterized by high resolution NMR $\left({ }^{1} \mathrm{H},{ }^{13} \mathrm{C},{ }^{31} \mathrm{P}\right)$, mass spectrometry and liquid chromatography mass spectrometry (LC-MS).

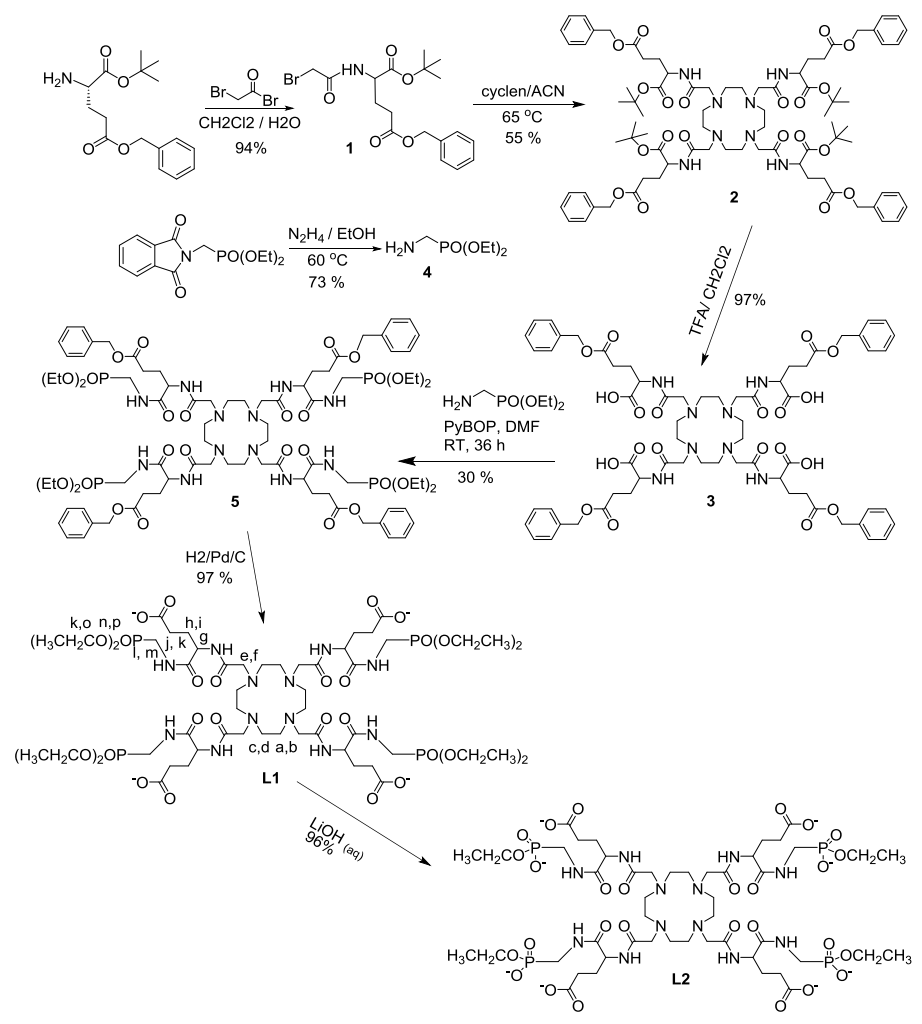

Scheme S1. Synthetic scheme for preparation of ligands L1 and L2 


\section{SYNTHETIC METHODS}

2-(2-Bromo-acetylamino)-pentanedioic acid 5-benzyl ester 1-tert-butyl ester (1). 2-Amino-pentanedioic acid 5-benzyl ester 1-tert-butyl ester (3.0 g, $9.1 \mathrm{mmol})$ and potassium carbonate $(3.77 \mathrm{~g}, 22.7 \mathrm{mmol})$ were dissolved in dichloromethane $(200 \mathrm{ml})$ and water $(100 \mathrm{~mL})$. The mixture was cooled to $\mathrm{o}{ }^{\circ} \mathrm{C}$, and a solution of 2 - bromoacetylbromide $(0.95 \mathrm{ml}$, $11.0 \mathrm{mmol}$ ) was added drop-wise with stirring over $30 \mathrm{~min}$. The reaction mixture was stirred at room temperature for $16 \mathrm{~h}$ then transferred to a separatory funnel to separate the two phases; the organic layer was washed with water $(2 \times 50 \mathrm{~mL})$ followed by brine $(2 \times$ $50 \mathrm{~mL})$. The organic extract was dried $\left(\mathrm{Na}_{2} \mathrm{SO}_{4}\right)$ and the solvents were removed under vacuum to afford the title compound as a colorless oil (Yield 3.54 g, 94\%). ${ }^{1} \mathrm{H}$ NMR (400 $\left.\mathrm{MHz}, \mathrm{CDCl}_{3}\right): \delta(\mathrm{ppm}) 1.48(9 \mathrm{H}, \mathrm{s}), 2.03\left(\mathrm{1H}, \mathrm{m},{ }^{3} \mathrm{~J} \mathrm{H}_{\mathrm{H}}=8.6 \mathrm{~Hz}\right), 2.26\left(\mathrm{1H}, \mathrm{m},{ }^{3} \mathrm{~J} \mathrm{H}-\mathrm{H}=6.7\right.$ $\mathrm{Hz}), 2.45\left(2 \mathrm{H}, \mathrm{m},{ }^{3} \mathrm{~J}_{\mathrm{H}-\mathrm{H}}=7.9 \mathrm{~Hz}\right), 3.85(2 \mathrm{H}, \mathrm{s}), 4.52\left(\mathrm{lH}, \mathrm{m},{ }^{3} J_{\mathrm{H}-\mathrm{H}}=5.0 \mathrm{~Hz}\right), 7.14\left(1 \mathrm{H}, \mathrm{d},{ }^{3} \mathrm{~J}\right.$ $\mathrm{H}-\mathrm{H}=7.6, \mathrm{NH}), 7.37\left({ }_{5} \mathrm{H}, \mathrm{m}, \mathrm{Ph}\right) .{ }^{13} \mathrm{C}$ NMR $\left(400 \mathrm{MHz}, \mathrm{CDCl}_{3}\right): \delta=27.36\left(-\mathrm{CH}_{2}-\right), 27.97$ $\left(\mathrm{C}\left(\mathrm{CH}_{3}\right)_{3}\right), \quad 28.74 \quad\left(\mathrm{CH}_{2} \mathrm{CH}_{2} \mathrm{CO}\right), \quad 30.16 \quad\left(\mathrm{BrCH}_{2} \mathrm{CO}\right), \quad 52.77 \quad\left(\mathrm{NH} \underline{\mathrm{CH}}\left(\mathrm{CH}_{2}\right)(\mathrm{CO})\right), 66.61$ $\left(\mathrm{OCH}_{2} \mathrm{Ph}\right), 82.94\left(\underline{\mathrm{C}}\left(\mathrm{CH}_{3}\right)_{3}\right), 128.3$ (2-Ph), $128.35(1-\mathrm{Ph}), 128.6$ (2-Ph), $135.7(1-\mathrm{Ph}), 165.59$ $\left(\mathrm{BrCH}_{2} \mathrm{CO}\right), 170.29\left(\mathrm{OCOCH}_{2}\right), 172.5(\mathrm{CH} \underline{\mathrm{COO}})$.

2-(2-\{4,7,10-Tris-[(3-benzyloxycarbonyl-1-tert-butoxycarbonyl -propylcarbamoyl)methyl]-1,4,7,1otetraaza-cyclododec-1-yl\}-acetylamino)-pentanedioic acid 5-benzyl ester 1-tert-butyl ester (2). 1,4,7,10-tetraazacyclododecane (1.2 g, $6.9 \mathrm{mmol})$ and potassium carbonate $(5 \mathrm{~g}, 7.1 \mathrm{~mol})$ were dissolved in anhydrous acetonitrile $(200 \mathrm{~mL})$ with stirring under $\mathrm{N}_{2}$ for 10 min followed by drop-wise addition of $1(13.2 \mathrm{~g}, 31.8 \mathrm{mmol})$ at ambient temperature over $30 \mathrm{~min}$. The reaction mixture was then stirred at $65^{\circ} \mathrm{C}$ for $20 \mathrm{~h}$ before the solvent was removed under vacuum. The resulting product was dissolved in dichloromethane $(100 \mathrm{~mL})$ and transferred to a separatory funnel; the organic layer was washed with water $(2 \times 50 \mathrm{~mL})$ followed by brine $(2 \times 50 \mathrm{~mL})$, dried over anhydrous $\mathrm{Na}_{2} \mathrm{SO}_{4}$. The solvent was removed under reduced pressure to yield an oily product which was purified by reverse phase HPLC on a $\mathrm{C}_{1} 8$ column using solvent gradient conditions to obtain the desired product as a pale yellow hygroscopic solid. (Yield $5.76 \mathrm{~g}, 55 \%$ ). Melting point $(\mathrm{Mp})=61-62{ }^{\circ} \mathrm{C} .{ }^{1} \mathrm{H}$ NMR $\left(400 \mathrm{MHz}, \mathrm{CDCl}_{3}\right): \delta(\mathrm{ppm}) 1.45(36 \mathrm{H}, \mathrm{s}), 1.98\left(4 \mathrm{H}, \mathrm{m},{ }^{3} \mathrm{~J}_{\mathrm{H}-}\right.$ $\mathrm{H}=8.8 \mathrm{~Hz}), 2.20\left(4 \mathrm{H}, \mathrm{m},{ }^{3} \mathrm{~J}_{\mathrm{H}-\mathrm{H}}=6.5 \mathrm{~Hz}\right), 2.43\left(8 \mathrm{H}, \mathrm{m},{ }^{3} \mathrm{~J}_{\mathrm{H}-\mathrm{H}}=7.42 \mathrm{~Hz}\right), 2.75(16 \mathrm{H}, \mathrm{br}$, ring $\left.\mathrm{CH}_{2}\right), 3.17(8 \mathrm{H}, \mathrm{s}), 4.45\left(4 \mathrm{H}, \mathrm{m},{ }^{3} \mathrm{~J}_{\mathrm{H}-\mathrm{H}}=7.6 \mathrm{~Hz}\right), 5.10(8 \mathrm{H}, \mathrm{s}), 7.35(5 \mathrm{H}, \mathrm{m}, \mathrm{Ph}), 7.6 \mathrm{o}(4 \mathrm{H}, \mathrm{br}$, $\left.{ }^{3} J_{\mathrm{H}-\mathrm{H}}=6.6 \mathrm{~Hz}, \mathrm{NH}\right) \cdot{ }^{13} \mathrm{C} \mathrm{NMR}\left(400 \mathrm{MHz}, \mathrm{CDCl}_{3}\right): \delta=27.39\left(\mathrm{CH}_{2} \mathrm{CH}_{2} \mathrm{CO}\right)$, 28.o $\left(\mathrm{C}\left(\mathrm{CH}_{3}\right)_{3}\right)$, $30.57\left(\mathrm{CH}_{2} \mathrm{CH}_{2} \mathrm{CO}\right)$, 52.40 $\left(\mathrm{CH}_{2}\right.$, br, ring), $58.84\left(\mathrm{NCH}_{2} \mathrm{CO}\right), 66.43\left(\mathrm{NHCH}\left(\mathrm{CH}_{2}\right)(\mathrm{CO})\right)$, 66.61 $\left(\mathrm{OCL}_{2} \mathrm{Ph}\right), 82.21\left(\underline{\mathrm{C}}\left(\mathrm{CH}_{3}\right)_{3}\right), 128.17(2-\mathrm{Ph}), 128.29(1-\mathrm{Ph}), 128.61(2-\mathrm{Ph}), 135.7(1-\mathrm{Ph})$, $171(2 \mathrm{CONH}, \mathrm{br}), 172.41$ (COO, br).

2-(2-\{4,7,10-Tris-[(3-benzyloxycarbonyl-1-carboxy-propylcarbamoyl)-methyl]1,4,7,10tetraaza-cyclododec-1-yl\}-acetylamino)-pentanedioic acid 5-benzyl ester (3). 
A solution of $2(0.5 \mathrm{~g}, 0.33 \mathrm{mmol})$ in dichloromethane $(2 \mathrm{~mL})$ and trifluoroacetic acid (TFA) ( $4 \mathrm{~mL}$ ) was stirred at room temperature for $24 \mathrm{~h}$. TFA was then removed in vacuo and the oily residue was dissolved in diethyl ether $(20 \mathrm{~mL})$ and stirred for $30 \mathrm{~min}$. The white precipitate that formed was filtered and washed with cold diethyl ether $(2 \times 20 \mathrm{~mL})$. The solid precipitate was dried then dissolved in water $(20 \mathrm{~mL})$ and lyophilized (Yield $0.41 \mathrm{~g}, 97$ \%). Melting point $(\mathrm{Mp})=132-133{ }^{\circ} \mathrm{C} .{ }^{1} \mathrm{H}$ NMR $(400 \mathrm{MHz}, \mathrm{MeOD}): \delta(\mathrm{ppm}) 2.02\left(4 \mathrm{H}, \mathrm{m},{ }^{3} \mathrm{~J}\right.$ $\mathrm{H}-\mathrm{H}=6.6 \mathrm{~Hz}), 2.20\left({ }_{4} \mathrm{H}, \mathrm{m},{ }^{3} \mathrm{H}-\mathrm{H}=7.7 \mathrm{~Hz}\right), 2.5 \mathrm{O}\left(8 \mathrm{H}, \mathrm{m},{ }^{3} \mathrm{~J}-\mathrm{H}=7.5 \mathrm{~Hz}\right), 3.2(16 \mathrm{H}, \mathrm{br}$, ring $\left.\mathrm{CH}_{2}\right), 3.7(8 \mathrm{H}, \mathrm{br}), 4.46(4 \mathrm{H}, \mathrm{br}), 5.09(8 \mathrm{H}, \mathrm{s}), 7.32(5 \mathrm{H}, \mathrm{m}, \mathrm{Ph}) \cdot{ }^{13} \mathrm{C}$ NMR (40o MHz, $\left.\left.\mathrm{CDCl}_{3}\right): \delta=26.26 \mathrm{CH}_{2} \mathrm{CH}_{2} \mathrm{CO}\right)$, $30.12\left(\mathrm{CH}_{2} \mathrm{CH}_{2} \mathrm{CO}\right)$, $50.5\left(\mathrm{CH}_{2}\right.$, br, ring), $51.88\left(\mathrm{NCH}_{2} \mathrm{CO}\right)$ $54.88\left(\mathrm{NH} \underline{\mathrm{CH}}\left(\mathrm{CH}_{2}\right)(\mathrm{CO})\right), 66.05\left(\mathrm{OCH}_{2} \mathrm{Ph}\right), 127.83(2-\mathrm{Ph}), 127.94(1-\mathrm{Ph}), 128.2(2-\mathrm{Ph}), 136.14$ (1-Ph), 172.84 (3CO, br). ESI-MS m/z: [M + H] ${ }^{+}$Calcd. for $\mathrm{C}_{64} \mathrm{H}_{81} \mathrm{~N}_{8} \mathrm{O}_{20}$ 1281.5; Found 1281.5. Aminomethyl-phosphonic acid diethyl ester (4). The synthesis of compound 4 was performed using previously reported methods. ${ }^{1,2}{ }^{1} \mathrm{H}$ NMR $\left(400 \mathrm{MHz}, \mathrm{CDCl}_{3}\right): \delta 1.3(6 \mathrm{H}, \mathrm{t}$, $\left.{ }^{3} \mathrm{~J}_{\mathrm{H}-\mathrm{H}}=7\right), 2.07\left(2 \mathrm{H}, \mathrm{s} \mathrm{br}, \mathrm{NH}_{2}\right), 3.00\left(2 \mathrm{H}, \mathrm{d},{ }^{2} \mathrm{~J}_{\mathrm{H}-\mathrm{P}}=10 \mathrm{~Hz}, \mathrm{NCH}_{2} \mathrm{P}\right), 4.14\left(4 \mathrm{H}, \mathrm{m},{ }^{3} \mathrm{~J}_{\mathrm{H}-\mathrm{H}}=7\right.$ $\mathrm{Hz},) .{ }^{31} \mathrm{P} \mathrm{NMR}\left(400 \mathrm{MHz}, \mathrm{CDCl}_{3}\right): 27.60(\mathrm{PP}, \mathrm{s})$.

tetrabenzyl 4,4',4",4"'-((2,2',2",2"'-(1,4,7,10-tetraaza cyclo dodecane-1,4,7,10tetrayl)tetrakis (acetyl))tetrakis (azanediyl))tetrakis(5-((diethoxyphosphoryl) methyl) amino)-5-oxopentanoate) (5).

Compound 3 (o.29 g , o.23 mmol), compound 4 (o.22 g,1.32 mmol), PyBOP (Benzotriazol-1yloxy)tripyrrolidino phosphor nium hexafluorophosphate) (o.69 g,1.33 mmol) and DIPEA $(557 \mu \mathrm{L}, 3.21 \mathrm{mmol})$ were dissolved in dry DMF $(5 \mathrm{~mL})$ and stirred at room temperature under a $\mathrm{N}_{2}$ atmosphere for 2 days. The solvent was removed under reduced pressure and the oily residue was purified by reverse phase HPLC on a $C_{1} 8$ column using solvent gradient (water/acetonitrile) conditions. Desired fractions were collected and solvents were evaporated and then lyophilized to give 5 , a yellow hygroscopic solid $(0.13 \mathrm{~g}, 30 \%) .{ }^{1} \mathrm{H}$ NMR (40o MHz, CDCl $): \delta(\mathrm{ppm}) 1.28\left(24 \mathrm{H}, \mathrm{m},{ }^{3} \mathrm{~J}_{\mathrm{H}-\mathrm{H}}=7 \mathrm{~Hz}\right), 2.06(4 \mathrm{H}, \mathrm{m}, \mathrm{br}), 2.19(4 \mathrm{H}, \mathrm{m}$, br), $2.51(8 \mathrm{H}, \mathrm{m}, \mathrm{br}), 3.2\left(16 \mathrm{H}\right.$, br, ring $\left.\mathrm{CH}_{2}\right), 3.7(8 \mathrm{H}, \mathrm{br}), 4.08\left(16 \mathrm{H}, \mathrm{m},{ }^{3} \mathrm{~J}_{\mathrm{H}-\mathrm{H}}=10 \mathrm{~Hz}\right), 4.42$ $\left({ }_{4} \mathrm{H}, \mathrm{m}, \mathrm{br}\right), 5.10(8 \mathrm{H}, \mathrm{s}), 7.33\left({ }_{5} \mathrm{H}, \mathrm{m}, \mathrm{br}, \mathrm{Ph}\right), 8.2\left({ }_{4} \mathrm{H}, \mathrm{br}, \mathrm{CON} \underline{\mathrm{HCH}}\right), 8.75\left({ }_{4} \mathrm{H}, \mathrm{br}\right.$, $\left.\mathrm{CONHCH}_{2}\right) \cdot{ }^{31} \mathrm{P}$ NMR $\left(400 \mathrm{MHz}, \mathrm{CDCl}_{3}\right): 22.67(1 \mathrm{P}, \mathrm{s}) .{ }^{13} \mathrm{C} \mathrm{NMR}\left(400 \mathrm{MHz}, \mathrm{CDCl}_{3}\right): \delta=$ 16.31 $\left(\mathrm{CH}_{3} \mathrm{CH}_{2} \mathrm{PO}\right), \quad 26.76 \quad\left(\mathrm{CHCH}_{2} \mathrm{CH}_{2}\right), \quad 30.51 \quad\left(\mathrm{CHCH}_{2} \mathrm{CH}_{2}\right), 35.39 \quad\left(\mathrm{~d}, \quad{ }^{1} \mathrm{~J}_{\mathrm{C}-\mathrm{p}=}{ }_{15} 6 \mathrm{~Hz}\right.$, $\mathrm{NHCH} 2 \mathrm{PO}$ ), 51.0 (ring $\mathrm{CH} 2), 53.38\left(\underline{\mathrm{CHCH}}_{2} \mathrm{CH}_{2}\right), 62.83\left(\mathrm{CH}_{3} \mathrm{CH}_{2} \mathrm{PO}\right), 66.41\left(\mathrm{OCH}_{2} \mathrm{Ph}\right)$, 128.15 (2-Ph), $128.26(1-\mathrm{Ph}), 128.57$ (2-Ph), $135.84(1-\mathrm{Ph}), 171.70 \quad\left(\mathrm{CH}_{2} \underline{\mathrm{CONH}}\right), 172.68$ $(\mathrm{CHCONH}), 172.86\left(\mathrm{CH}_{2} \mathrm{COO}\right)$. ESI-MS m/z: $[\mathrm{M}+\mathrm{Na}]^{+}$Calcd. for $\mathrm{C}_{84} \mathrm{H}_{12} 8 \mathrm{~N}_{12} \mathrm{NaO}_{2} 8 \mathrm{P}_{4}$ 1900.9; Found 1901.3.

4-[(Diethoxy-phosphorylmethyl)-carbamoyl]-4-\{2-[4,7,10-tris-(\{3-carboxy-1[(diethoxy-phosphorylmethyl)-carbamoyl]-propylcarbamoyl $\}$-methyl)1,4,7,1otetraaza-cyclododec-1-yl]-acetylamino\}-butyric acid (L1).

A solution of 5 (o.098g, $0.052 \mathrm{mmol}$ ) in ethanol was mixed with $15 \% \mathrm{Pd} / \mathrm{C}$ and stirred for 2 days under a $\mathrm{H}_{2}$ atmosphere. The solution was filtered through a syringe filter to remove $\mathrm{Pd} / \mathrm{C}$ and the solvent evaporated. The compound was dissolved in water and lyphophilized to obtain L1 (o.077g, 97\%). Mp = 81-82 ${ }^{\circ} \mathrm{C} .1 \mathrm{H}$ NMR $\left(400 \mathrm{MHz}, \mathrm{D}_{2} \mathrm{O}\right): \delta(\mathrm{ppm}) 1.25(24 \mathrm{H}, \mathrm{m}$, 
$\left.{ }^{3} \mathrm{~J}_{\mathrm{H}-\mathrm{H}}=7.1 \mathrm{~Hz}\right), 1.95\left(4 \mathrm{H}, \mathrm{m},{ }^{3} \mathrm{~J}_{\mathrm{H}-\mathrm{H}}=7.21 \mathrm{~Hz}\right), 2.05\left(4 \mathrm{H}, \mathrm{m},{ }^{3} \mathrm{~J}_{\mathrm{H}-\mathrm{H}}=6.26 \mathrm{~Hz}\right), 2.42\left(8 \mathrm{H}, \mathrm{t},{ }^{3} \mathrm{~J}_{\mathrm{H}-\mathrm{H}}=\right.$ $7.73 \mathrm{~Hz}), 3.26\left(16 \mathrm{H}\right.$, br, ring $\left.\mathrm{CH}_{2}\right), 3.65(8 \mathrm{H}, \mathrm{br}), 3.8\left(8 \mathrm{H}, \mathrm{dd},{ }^{3} \mathrm{~J}_{\mathrm{H}-\mathrm{H}}=6 \mathrm{~Hz},{ }^{2} \mathrm{~J}_{\mathrm{H}-\mathrm{P}}=12\right.$ $\left.\mathrm{Hz}, \mathrm{NCH}_{2} \mathrm{P}\right), 4.11\left(16 \mathrm{H}, \mathrm{m},{ }^{3} \mathrm{~J}_{\mathrm{H}-\mathrm{H}}=7.4 \mathrm{~Hz}\right), 4.36(4 \mathrm{H}, \mathrm{m}, \mathrm{br})$. ESI-MS m/z: $[\mathrm{M}+\mathrm{H}]^{+}$Calcd. for $\mathrm{C}_{56} \mathrm{H}_{105} \mathrm{~N}_{12} \mathrm{O}_{28} \mathrm{P}_{4}$ 1517.6; Found 1517.2.

4-[(Ethoxy-hydroxy-phosphorylmethyl)-carbamoyl]-4-\{2-[4,7,10-tris-(\{3-carboxy-1[(ethoxy-hydroxy-phosphoryl methyl)-carbamoyl]-propylcarbamoyl\}-methyl)1,4,7,10-tetraaza-cyclododec-1-yl]-acetylamino\}-butyric acid (L2).

Compound L1 (o.05g, $0.033 \mathrm{mmol}$ ) and lithium hydroxide monohydrate (0.033g, $79 \mathrm{mmol}$ ) were dissolved in water and stirred for one day at room temperature. The resulting solution was lyphophilized to obtain L2. The completion of reaction was monitored by analytical HPLC. (o.044 g, 96\%). Mp $=80-81{ }^{\circ} \mathrm{C} .{ }^{1} \mathrm{H}$ NMR (40o MHz, $\left.\mathrm{D}_{2} \mathrm{O}\right): \delta(\mathrm{ppm}) 1.15$ $\left(12 \mathrm{H}, \mathrm{m},{ }^{3} \mathrm{~J}_{\mathrm{H}-\mathrm{H}}=7.1 \mathrm{~Hz}\right), 1.89\left(4 \mathrm{H}, \mathrm{m},{ }^{3} \mathrm{~J}_{\mathrm{H}-\mathrm{H}}=7.2 \mathrm{OHz}\right), 1.95\left(4 \mathrm{H}, \mathrm{m},{ }^{3} \mathrm{~J}_{\mathrm{H}-\mathrm{H}}=6.4 \mathrm{O} \mathrm{Hz}\right), 2.20$ $\left(8 \mathrm{H}, \mathrm{t},{ }^{3} \mathrm{~J}_{\mathrm{H}-\mathrm{H}}=7.2 \mathrm{O} \mathrm{Hz}\right), 2.6\left(16 \mathrm{H}, \mathrm{br}\right.$, ring $\left.\mathrm{CH}_{2}\right), 3.3\left(16 \mathrm{H}, \mathrm{m}, \mathrm{br}, \mathrm{NCH}_{2} \mathrm{CO}, \mathrm{NCH}_{2} \mathrm{P}\right), 3.87$ $\left(8 \mathrm{H}, \mathrm{m},{ }^{3} \mathrm{~J}_{\mathrm{H}-\mathrm{H}}=7.2 \mathrm{~Hz}\right), 4.30(4 \mathrm{H}, \mathrm{m}, \mathrm{br})$. ESI-MS m/z: $[\mathrm{M}+\mathrm{H}]^{+}$Calcd. for $\mathrm{C}_{48} \mathrm{H}_{89} \mathrm{~N}_{12} \mathrm{O}_{28} \mathrm{P}_{4}$ 1405.5; Found 1405.2.

Preparation of Lanthanide(III) Complexes. The Ln(III) complexes were prepared by mixing stoichiometric amounts of $\mathrm{LnCl}_{3}$ (aq) or $\mathrm{Ln}(\mathrm{OTf})_{3}$ (aq) with each ligand in water. The resulting solution was adjusted to $\mathrm{pH}=6$ and maintained at that $\mathrm{pH}$ by careful addition of $1 \mathrm{M} \mathrm{NaOH}$ and stirred at room temperature. Formation of complex was monitored by analytical HPLC. After completion, excess metal is filtered off after adjusting the $\mathrm{pH}$ of the solution to $\sim 8.5$. The solvent was removed under reduced pressure and the complexes were purified by HPLC. ESI-MS m/z: $[\mathrm{M}+\mathrm{H}]^{+}$Calcd. for $\mathrm{C}_{56} \mathrm{H}_{105} \mathrm{EuN}_{12} \mathrm{O}_{28} \mathrm{P}_{4}{ }^{3+}{ }_{1670.5}$; Found $1670.0 \mathrm{~m} / \mathrm{z}$; $\left[\mathrm{M}+\mathrm{H}^{+}\right]$Calcd. for $\mathrm{C}_{48} \mathrm{H}_{84} \mathrm{EuN}_{12} \mathrm{O}_{28} \mathrm{P}^{4-} 1554.4$ : Found $\left[\mathrm{M}+\mathrm{H}^{+}\right]^{+}: 1554.8$.

NMR SPECTRA

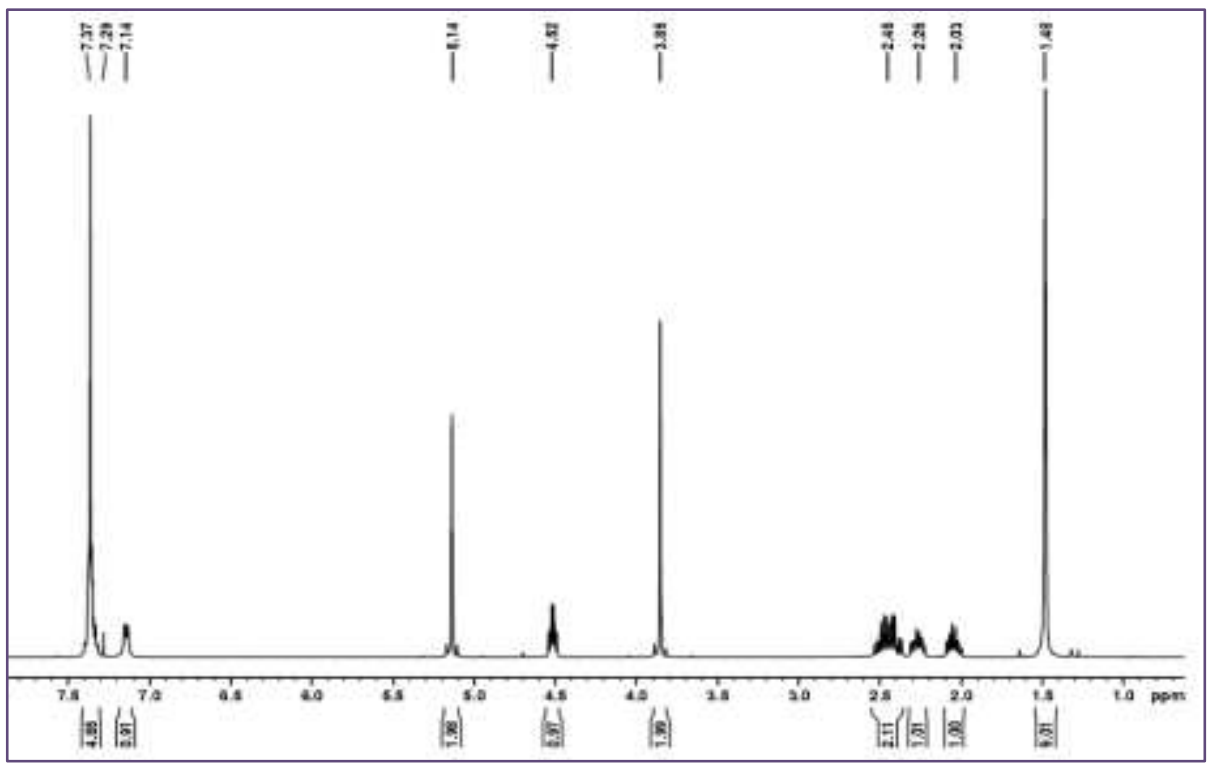


Figure A.1. ${ }^{1} \mathrm{H}$ NMR spectrum for compound 1 in $\mathrm{CDCl}_{3}$ (scheme $\mathrm{S} 1$ ).

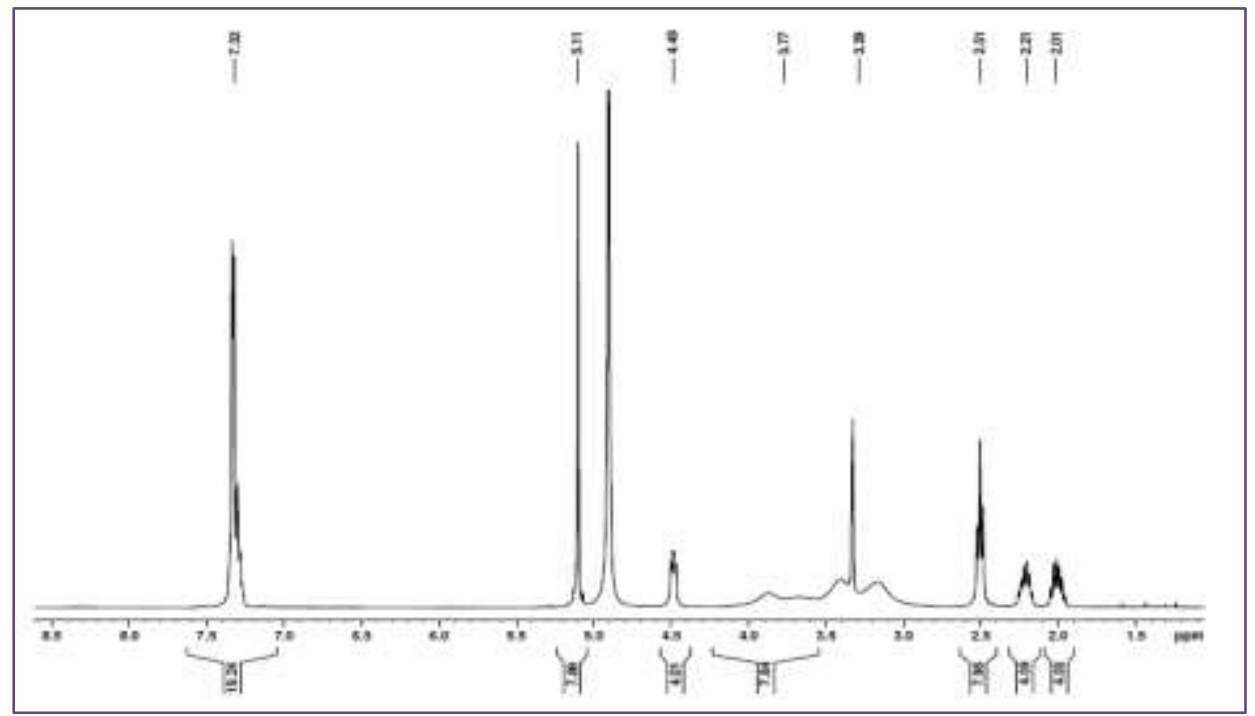

Figure A.2. $1 \mathrm{H}$ NMR spectrum for compound 3 in MeOD (scheme 1)
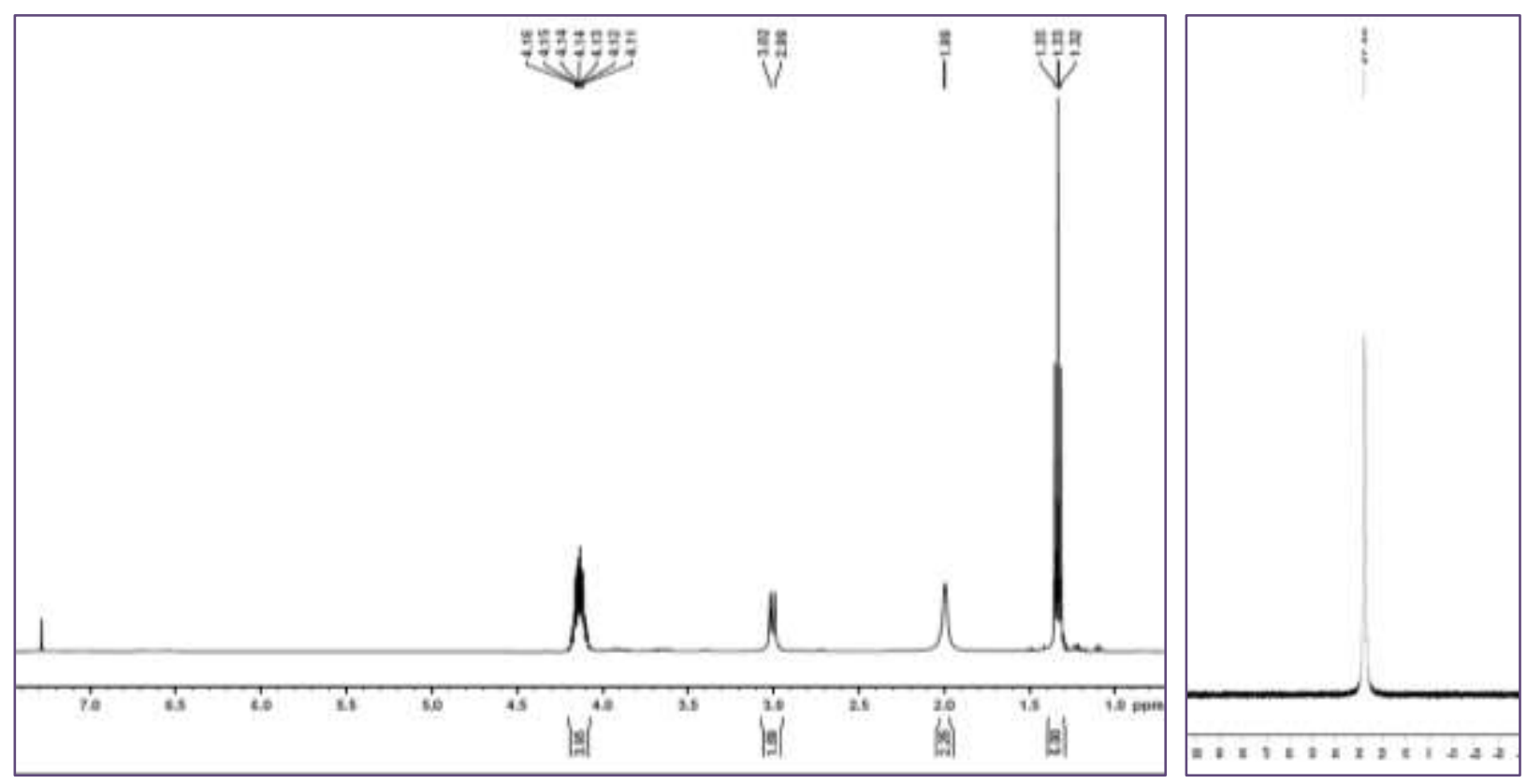
Figure A.3. Left- ${ }^{1} \mathrm{H}$ NMR spectrum for compound 4 in $\mathrm{CDCl}_{3}$. Right ${ }^{31} \mathrm{P}$ spectrum for 4 (scheme 1)

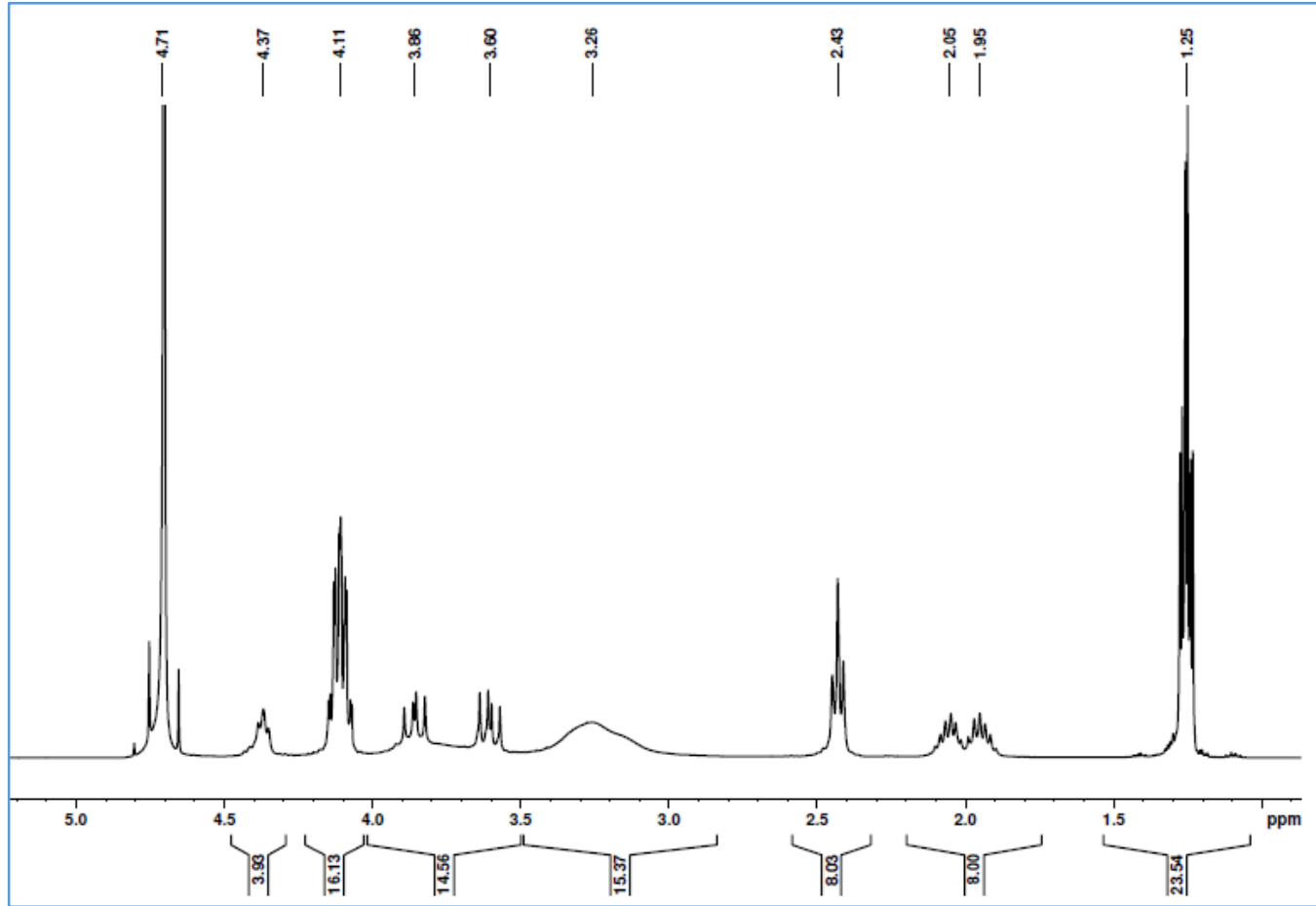

Figure A.4. ${ }^{1} \mathrm{H}$ NMR spectrum for $\mathrm{L} 1$ in $\mathrm{D}_{2} \mathrm{O}$ (scheme $\mathrm{S}_{1}$ )

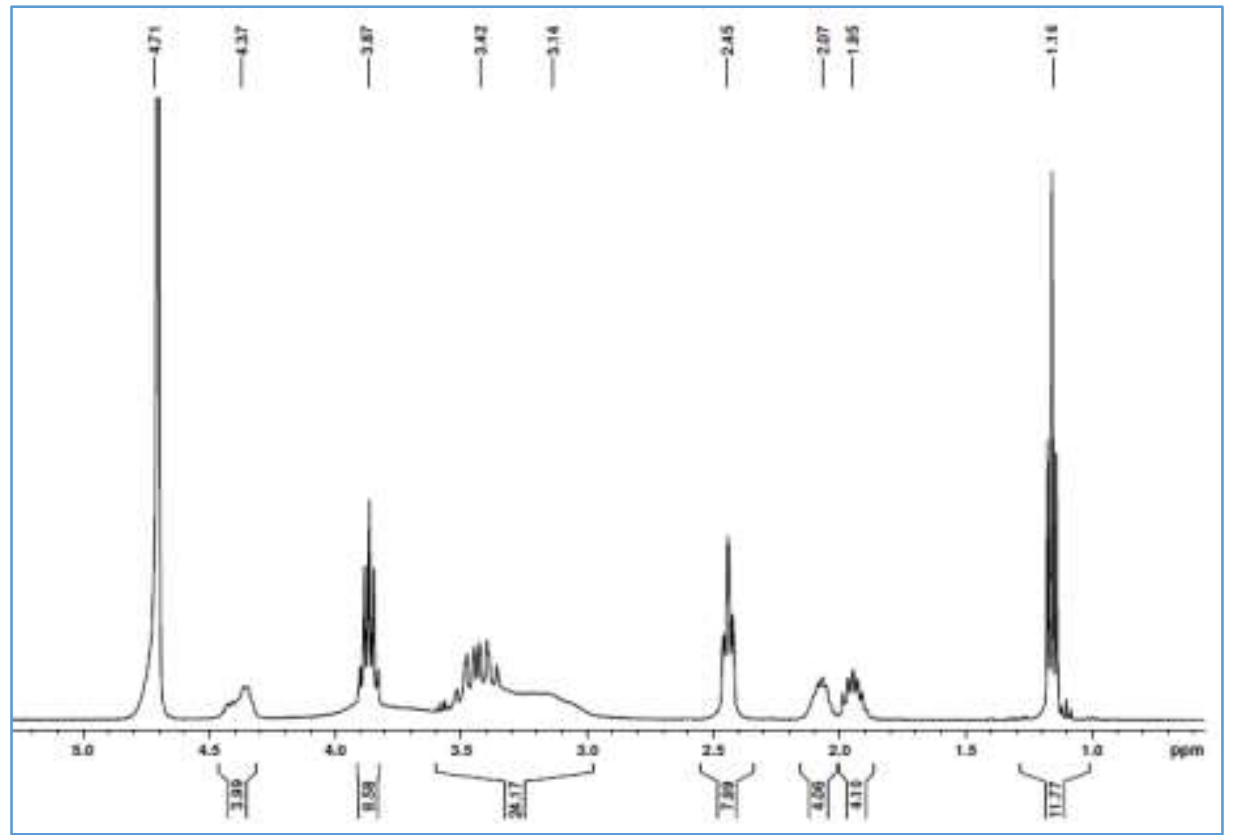


Figure A.5. ${ }^{1} \mathrm{H}$ NMR spectrum for $\mathrm{L}_{2}$ in $\mathrm{D}_{2} \mathrm{O}$ (scheme 1)

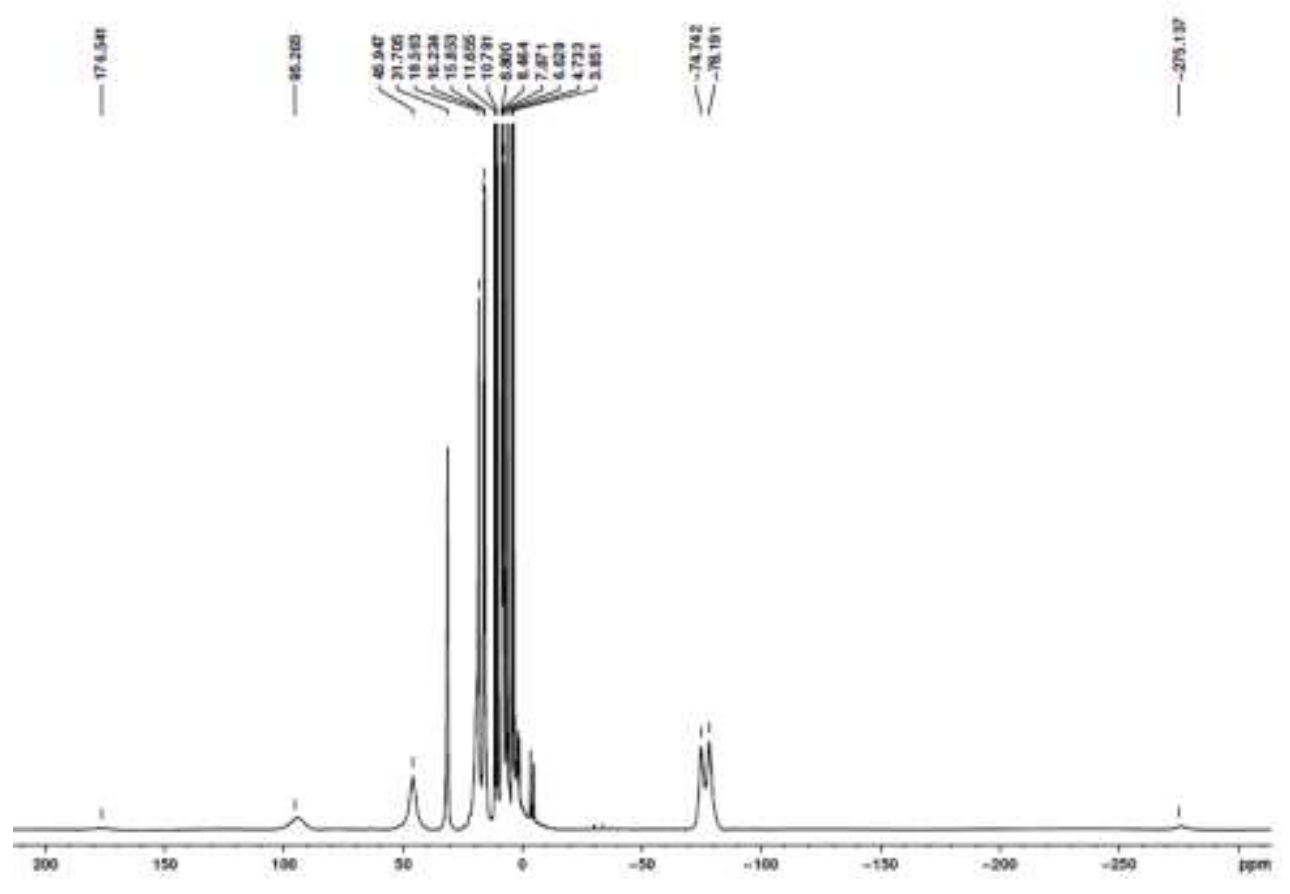

Figure A 6. $1 \mathrm{H}$ NMR of TbLi in $\mathrm{D}_{2} \mathrm{O}$ 


\section{HPLC PROFILES}

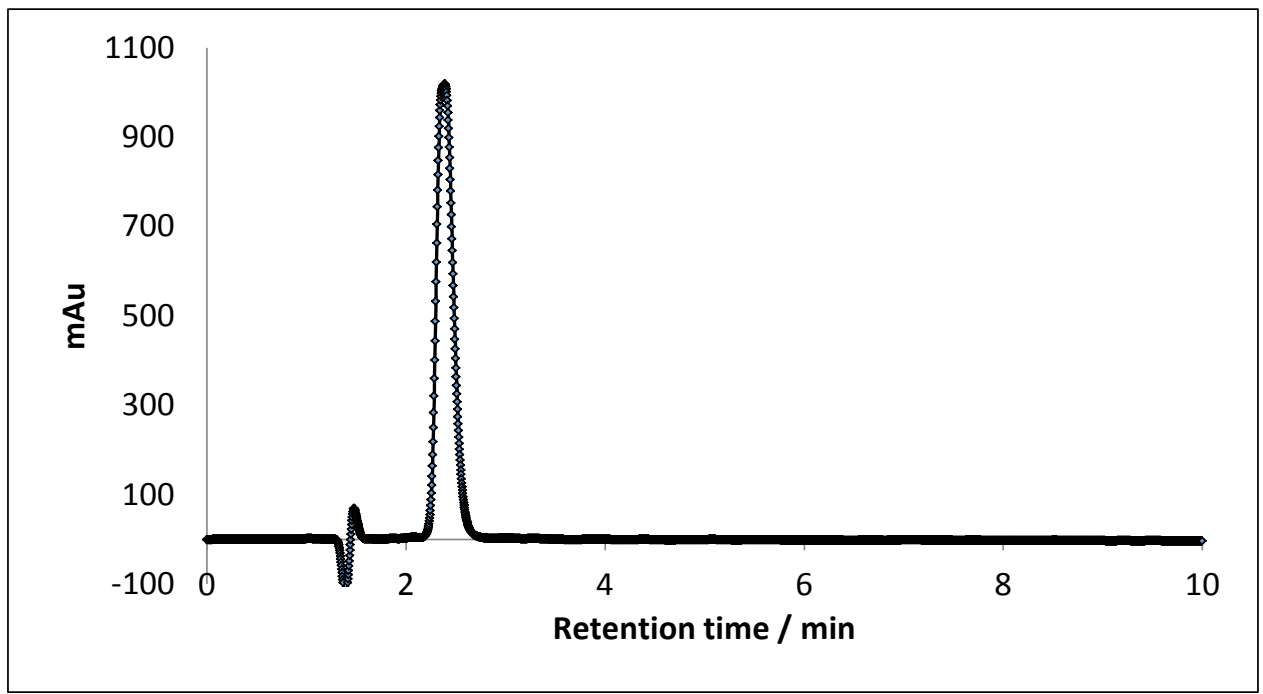

Figure B.1. Analytical HPLC profile for purified EuL1 complex; solvent gradient conditions- $80 \%$ Water (1\% TFA): $20 \%$ ACN to $50 \%$ water ( $1 \%$ TFA): $20 \%$ ACN in 180 min.

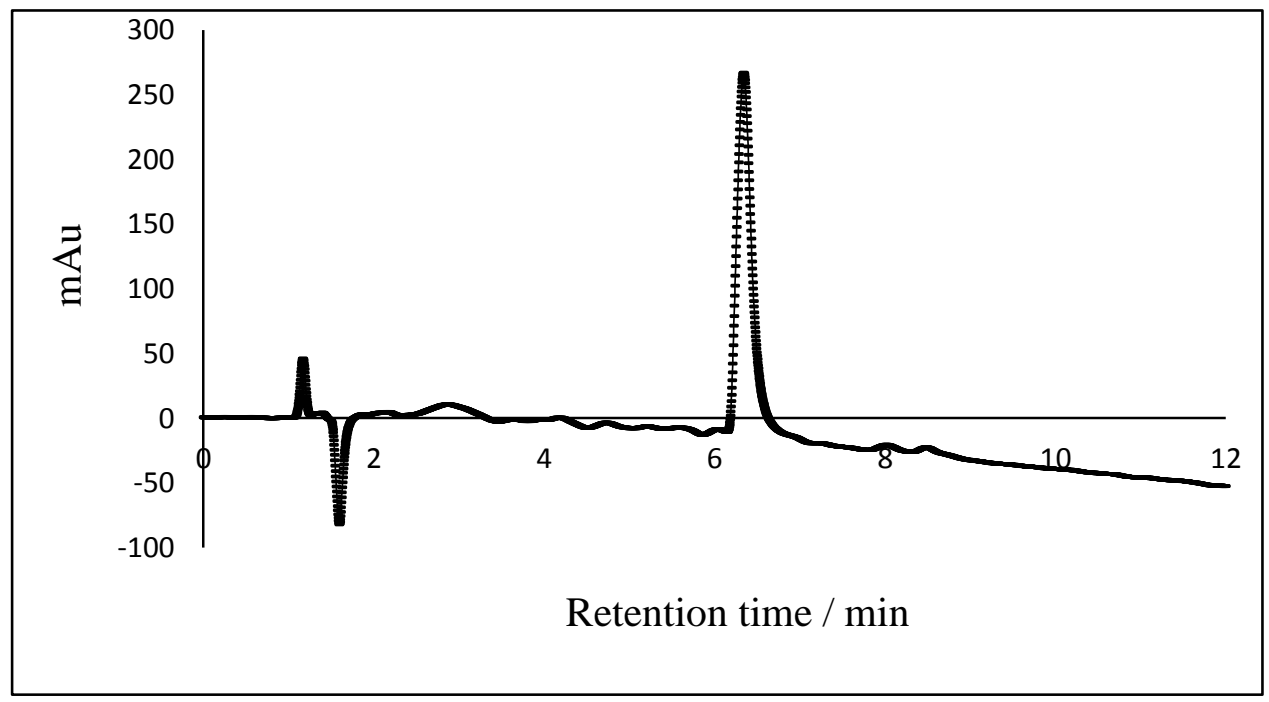

Figure B.2. Analytical HPLC profile for purified EuL2 complex; solvent gradient conditions-98\% Water ( $1 \%$ TFA): $2 \%$ ACN to $40 \%$ water ( $1 \%$ TFA): $60 \%$ ACN in 30 min. 


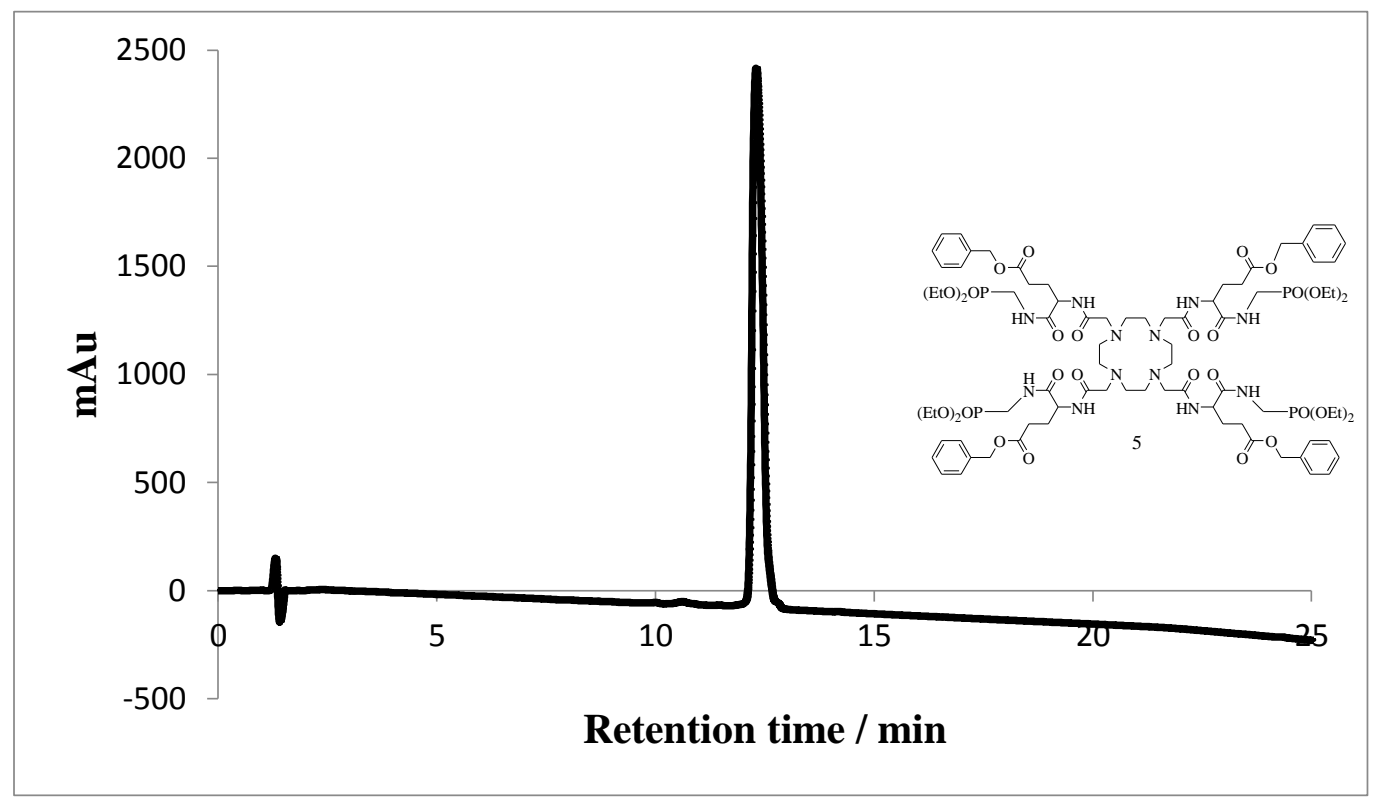

Figure B.3. Analytical HPLC profiles for purified compound 5 (scheme 1) solvent gradient conditions- $80 \%$ Water ( $1 \%$ TFA): $20 \% \mathrm{ACN}$ to $27 \%$ water ( $1 \%$ TFA): $73 \% \mathrm{ACN}$ in $20 \mathrm{~min}$. 


\section{CEST SPECTRA, STRUCTURE ELUCIDATION, COMPUTED POTENTIALS AND IN VIVO SUPPORTING INFORMATION}

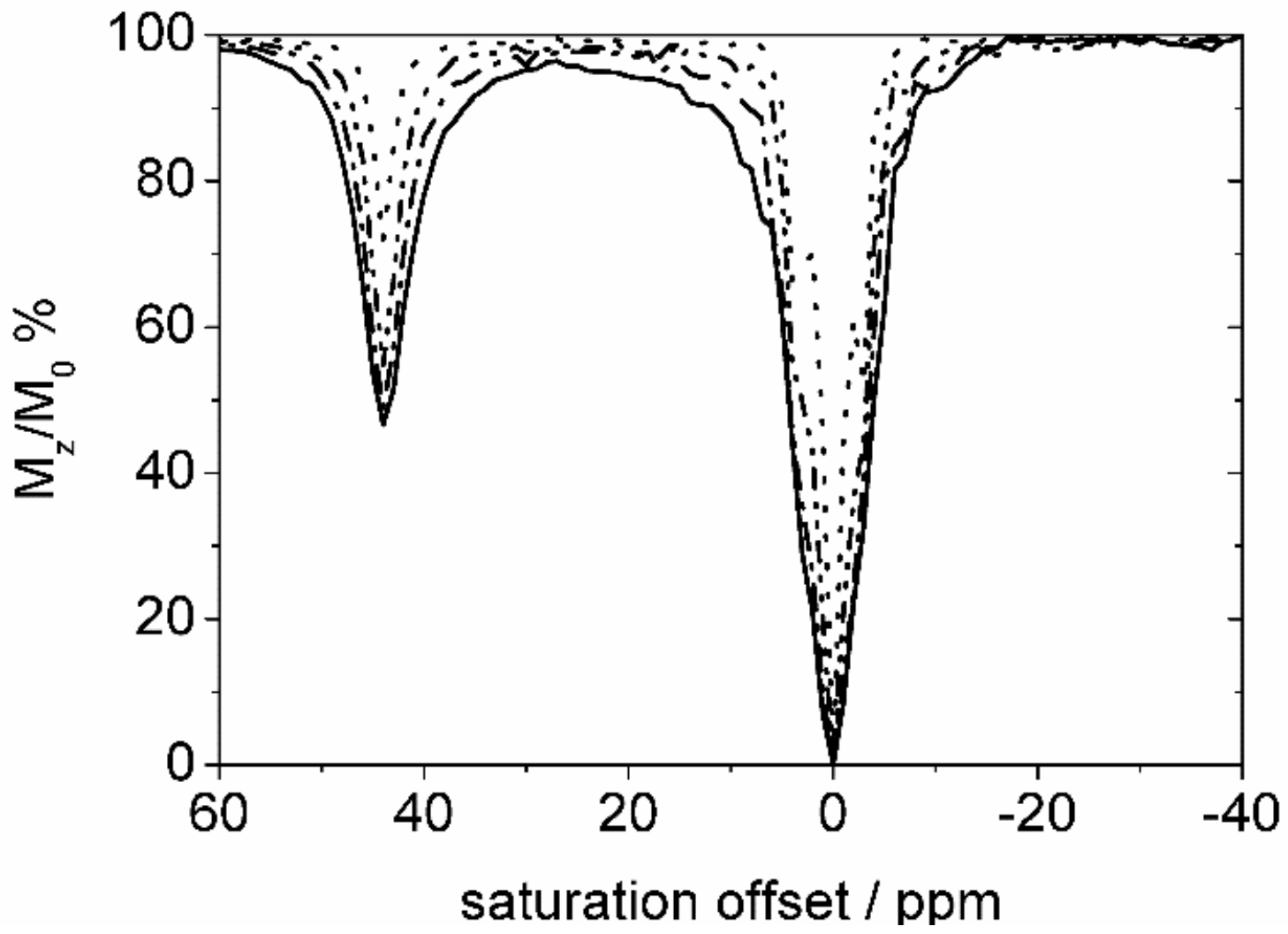

Figure C.1. CEST spectra of $30 \mathrm{mM}$ EuL1 collected with varying $B_{1}=4.7 \mu \mathrm{T}$ (dot lines), 9.4 $\mu \mathrm{T}$ (dash dot dot lines), 14.1 $\mathrm{T}$ (dash dot lines), $18.8 \mu \mathrm{T}$ (straight line) with a presaturation time of $7 \mathrm{~s}$ at $298 \mathrm{~K}$. 

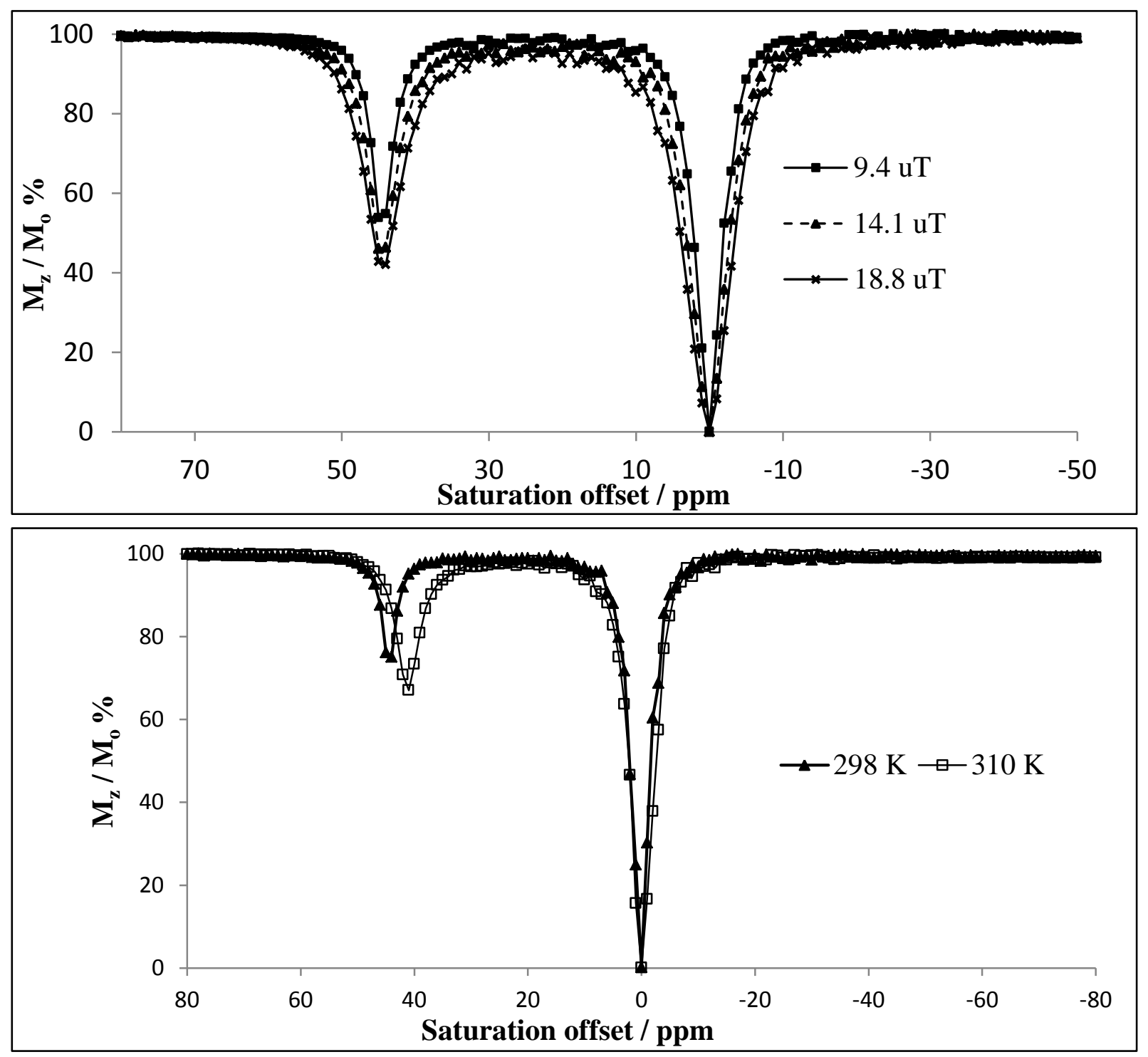

Figure C.2. (Top).CEST spectra of $40 \mathrm{mM}$ EuL2 collected with varying $\mathrm{B}_{1}=9.4 \mu \mathrm{T}, 14.1 \mu \mathrm{T}$, $18.8 \mu \mathrm{T}$ with a presaturation time of $7 \mathrm{~s}$ at $298 \mathrm{~K}$. (Bottom). CEST spectra for $14 \mathrm{mM} \mathrm{EuL2}$ at $\mathrm{pH} 7$ at $9.4 \mathrm{uT}$, saturation time $=5 \mathrm{~s}$. 


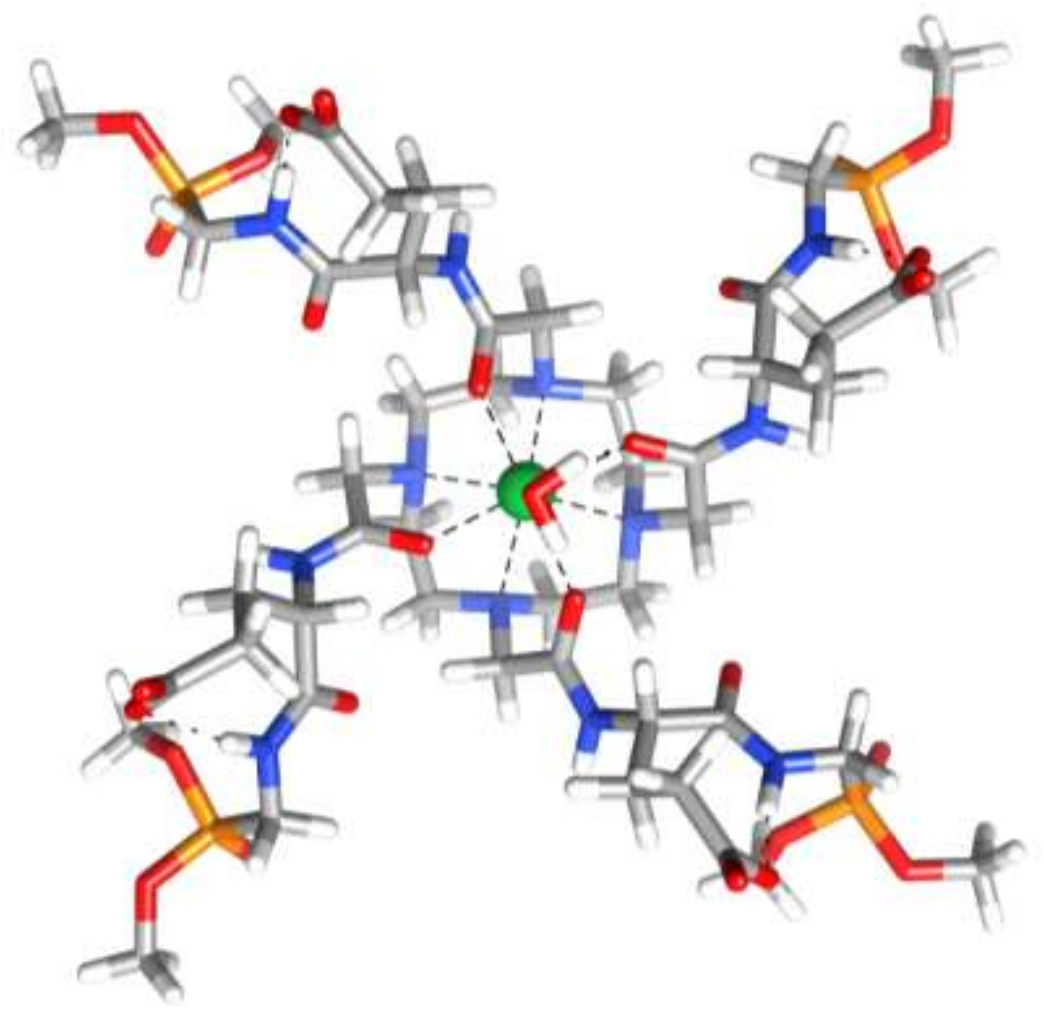

Figure C.3. Structure of the $\left[\mathrm{Yb}\left(\mathrm{L}^{\prime}{ }^{\prime}\right)\left(\mathrm{H}_{2} \mathrm{O}\right)\right]^{3+}$ complex optimized in aqueous solution at the TPSSh/LCRECP/6-31G(d) level. View along the pseudo $C_{4}$ symmetry axis. 


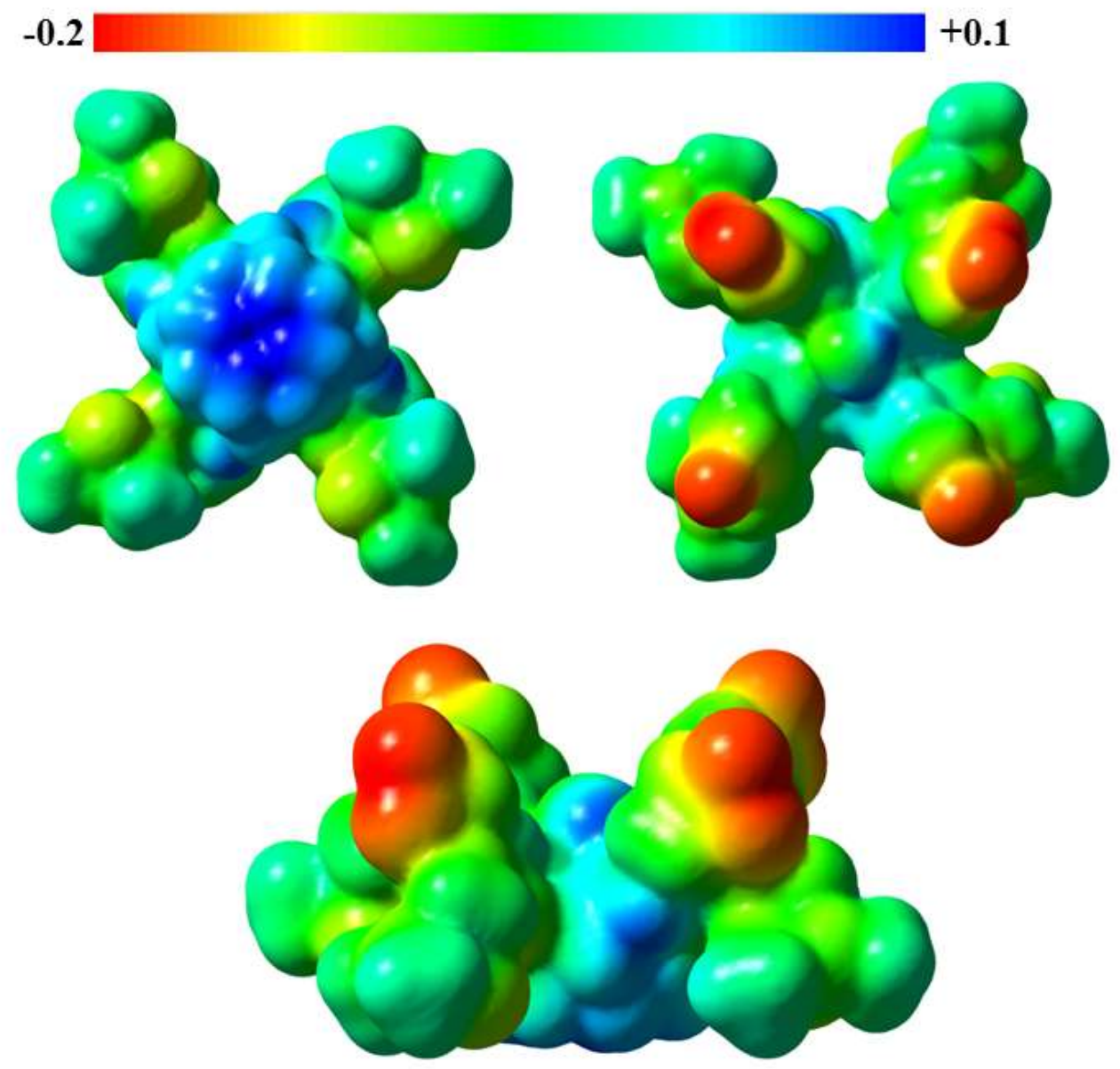

Figure C.4. Computed TPSSh/LCRECP/6-31G(d) electrostatic potential (hartree) for the Yb-2 complex on the molecular surface defined by the o.oor electrons $\cdot$ bohr $^{-3}$ contour of the electronic density. Top: Views along the pseudo $C_{4}$ symmetry axis. Bottom: Lateral view. 

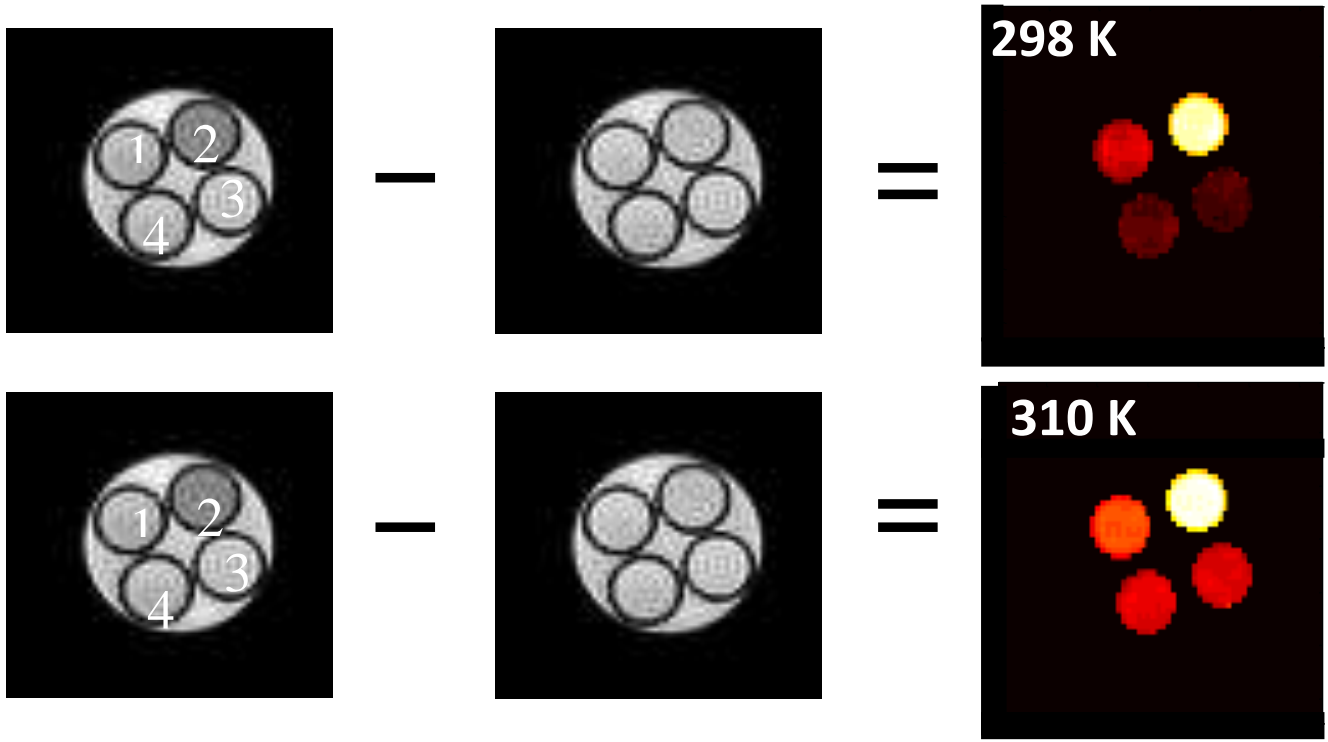

Figure C.5. Top - a) Proton intensity images acquired at a presaturation offset of $44 \mathrm{ppm}$ (left). b) -44 ppm (right) for EuL1 at $298 \mathrm{~K}$. Samples - 1: $20 \mathrm{mM}$ in plasma; 2: $20 \mathrm{mM}$ in water; 3: $10 \mathrm{mM}$ in water; 4: $10 \mathrm{mM}$ in plasma .c) Processed CEST image overlapping with proton intensity image acquired at $298 \mathrm{~K}$. Bottom - images acquired at $310 \mathrm{~K}$. Saturation power $=400 \mathrm{~Hz}$; saturation time $=5 \mathrm{~s}$.

Table C.1. ${ }^{1} \mathrm{H}$ NMR Shifts (ppm) for L1 complexes $(\mathrm{Ln}=\mathrm{Tb}, \mathrm{Yb})$ and comparison of the experimental and calculated paramagnetic ${ }^{1} \mathrm{H}$ NMR shifts for the $\mathrm{Yb}^{3+}$ complex.

\begin{tabular}{|c|c|c|c|c|}
\hline & $\mathrm{Tb}^{3+}$ & \multicolumn{2}{|c|}{$\mathrm{Yb}^{3+}$} & \\
\hline & $\delta_{i}^{\text {exp }}$ & $\delta_{i}^{\text {exp }}$ & $\delta_{i}^{\text {calca }}$ & $\left(3 \cos ^{2} \theta-1\right) / \mathrm{r}^{3}$ \\
\hline $\mathrm{a}(\mathrm{ax})$ & -275.1 & 80.9 & 77.8 & 0.02944 \\
\hline $\mathrm{b}(\mathrm{eq})$ & -78.2 & 15.2 & 15.6 & 0.00497 \\
\hline $\mathrm{c}(\mathrm{ax})$ & 95.3 & -25.4 & -23.5 & -0.10410 \\
\hline $\mathrm{d}(\mathrm{eq})$ & -74.7 & 12.56 & 14.4 & 0.00450 \\
\hline $\mathrm{e}(\mathrm{ax})$ & 176.5 & -45.5 & -50.2 & -0.02094 \\
\hline $\mathrm{f}(\mathrm{eq})$ & 46.0 & -20.6 & -19.6 & -0.00889 \\
\hline $\mathrm{g}$ & 18.6 & -3.4 & -4.2 & -0.00285 \\
\hline
\end{tabular}

${ }^{a}$ Labels as shown in Scheme $\mathrm{S}_{1}{ }^{b}$ Calculated chemical shifts obtained using the linear plot shown in Figure 7. 


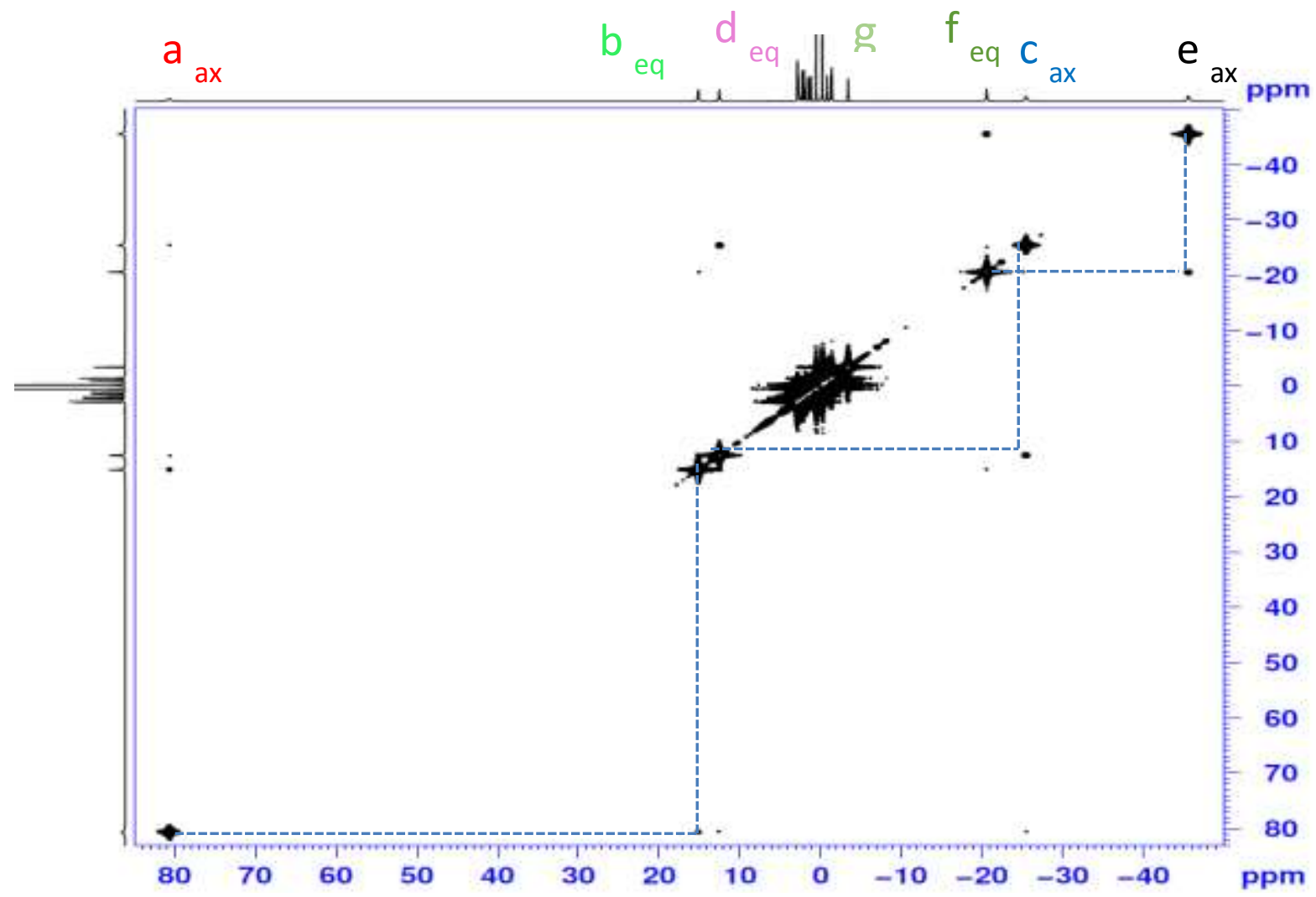

Figure C.6. ${ }^{1} \mathrm{H}-{ }^{1} \mathrm{H}$ NMR COSY NMR spectrum for YbLi in $\mathrm{D}_{2} \mathrm{O}$ 
a)

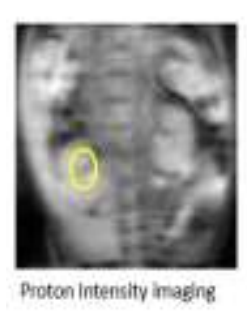

d)

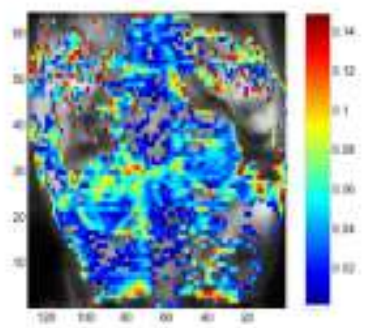

c)
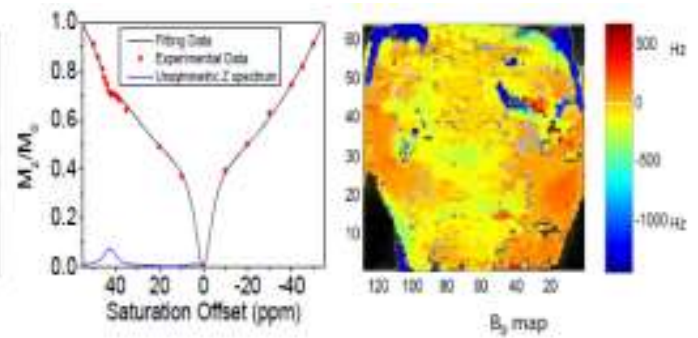

e)

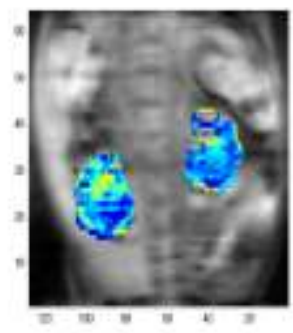

Figure C.7. Proton intensity image of EuL2. b) CEST/Z spectrum for ROI (yellow region) in left figure and corresponding fitting $\mathrm{Z}$ spectrum and unsymmetrical $\mathrm{Z}$. C) $\mathrm{B}_{\mathrm{o}}$ maps overlapping with proton intensity image. d) CEST image after 8 min post injection at 42 ppm. c) CEST image of kidneys after 8 min post injection at $42 \mathrm{ppm}$ overlap on the proton intensity image. $B_{1}=9 \cdot 4 \mu \mathrm{T}$.

\section{FURTHER COMPUTATIONAL DETAILS:}

Owing to the large size of the systems investigated and the slow convergence of the optimizations, they were stopped when the convergence parameters were about twice the default values. Thus, the final geometries correspond to stable conformations for the chosen minimization algorithm rather than true energy minima. For these reasons, frequency analysis was not carried out to characterize the stationary points. Optimized Cartesian coordinates $(\AA)$ :

Yb-2, TPSSh/LCRECP/6-31G(d), aqueous solution

\begin{tabular}{lrrr}
\hline $\mathrm{N}$ & -1.98090000 & 0.73530000 & -2.42850000 \\
$\mathrm{C}$ & -2.21710000 & -0.25870000 & -3.52620000 \\
$\mathrm{C}$ & -2.05250000 & -1.71070000 & -3.08300000
\end{tabular}




\begin{tabular}{|c|c|c|c|}
\hline $\mathrm{N}$ & -0.75400000 & -1.93950000 & -2.37150000 \\
\hline $\mathrm{C}$ & 0.32180000 & -2.18880000 & -3.38240000 \\
\hline C & 1.73670000 & -2.07800000 & -2.81530000 \\
\hline $\mathrm{N}$ & 1.98640000 & -0.75560000 & -2.15540000 \\
\hline C & 2.27920000 & 0.26570000 & -3.19420000 \\
\hline C & 2.07080000 & 1.72180000 & -2.7662000 \\
\hline $\mathrm{N}$ & 0.69400000 & 2.01640000 & -2.2565000 \\
\hline C & -0.24310000 & 2.23430000 & -3.39780000 \\
\hline C & -1.71400000 & 2.06630000 & -3.04670000 \\
\hline C & -3.18280000 & 0.85690000 & -1.56800000 \\
\hline C & -3.17830000 & -0.20390000 & -0.4817000 \\
\hline O & -2.09320000 & -0.60430000 & 0.0221000 \\
\hline C & -0.90250000 & -3.11990000 & -1.4920000 \\
\hline C & 0.14560000 & -3.09980000 & -0.3961000 \\
\hline 0 & 0.55370000 & -2.00880000 & 0.1002000 \\
\hline C & 3.16580000 & -0.86180000 & -1.2680000 \\
\hline C & 3.15640000 & 0.25450000 & -0.2479000 \\
\hline O & 2.04340000 & 0.69820000 & 0.1734000 \\
\hline C & 0.75950000 & 3.25090000 & -1.4448000 \\
\hline $\mathrm{C}$ & -0.30010000 & 3.23800000 & -0.3710000 \\
\hline O & -0.66440000 & 2.12860000 & 0.1083000 \\
\hline $\mathrm{H}$ & -1.50180000 & -0.01920000 & -4.3189000 \\
\hline $\mathrm{H}$ & -3.22160000 & -0.12420000 & -3.9536000 \\
\hline $\mathrm{H}$ & -2.86140000 & -2.02450000 & -2.4185000 \\
\hline $\mathrm{H}$ & -2.12020000 & -2.35280000 & -3.9729000 \\
\hline $\mathrm{H}$ & 0.16480000 & -1.46990000 & -4.1912000 \\
\hline $\mathrm{H}$ & 0.19860000 & -3.18440000 & -3.8332000 \\
\hline $\mathrm{H}$ & 1.94130000 & -2.85770000 & -2.0772000 \\
\hline $\mathrm{H}$ & 2.44340000 & -2.24390000 & -3.6403000 \\
\hline $\mathrm{H}$ & 1.66210000 & 0.02640000 & -4.0638000 \\
\hline $\mathrm{H}$ & 3.31850000 & 0.14850000 & -3.5332000 \\
\hline $\mathrm{H}$ & 2.77650000 & 2.01650000 & -1.9847000 \\
\hline $\mathrm{H}$ & 2.30040000 & 2.35280000 & -3.6368000 \\
\hline $\mathrm{H}$ & 0.04070000 & 1.54590000 & -4.1931000 \\
\hline $\mathrm{H}$ & -0.09910000 & 3.24790000 & -3.8002000 \\
\hline $\mathrm{H}$ & -2.06070000 & 2.84550000 & -2.3620000 \\
\hline $\mathrm{H}$ & -2.29960000 & 2.18230000 & -3.9696000 \\
\hline $\mathrm{H}$ & -3.12370000 & 1.83340000 & -1.0707000 \\
\hline $\mathrm{H}$ & -4.11370000 & 0.85140000 & -2.1526000 \\
\hline $\mathrm{H}$ & -1.89090000 & -3.05130000 & -1.0170000 \\
\hline $\mathrm{H}$ & -0.88390000 & -4.05960000 & -2.0615000 \\
\hline $\mathrm{H}$ & 3.10920000 & -1.82250000 & -0.7377000 \\
\hline $\mathrm{H}$ & 4.10330000 & -0.86240000 & -1.8402000 \\
\hline $\mathrm{H}$ & 1.73380000 & 3.25710000 & -0.9399000 \\
\hline $\mathrm{H}$ & 0.70750000 & 4.15950000 & -2.0612000 \\
\hline O & -0.02910000 & 0.02390000 & 1.7979000 \\
\hline $\mathrm{H}$ & 0.28530000 & -0.90360000 & 1.7985000 \\
\hline $\mathrm{H}$ & 0.84080000 & 0.47710000 & 1.7776000 \\
\hline
\end{tabular}




\begin{tabular}{|c|c|c|c|}
\hline $\mathrm{Yb}$ & -0.01920000 & 0.10010000 & -0.68850000 \\
\hline $\mathrm{N}$ & -0.76950000 & 4.39550000 & 0.08970000 \\
\hline $\mathrm{N}$ & 4.32310000 & 0.68220000 & 0.22150000 \\
\hline $\mathrm{N}$ & 0.57250000 & -4.25690000 & 0.08380000 \\
\hline $\mathrm{N}$ & -4.33660000 & -0.63900000 & 0.00190000 \\
\hline $\mathrm{H}$ & -0.52310000 & 5.24700000 & -0.40640000 \\
\hline $\mathrm{H}$ & 5.16460000 & 0.32850000 & -0.22480000 \\
\hline $\mathrm{H}$ & 0.28060000 & -5.10700000 & -0.39130000 \\
\hline $\mathrm{H}$ & -5.19710000 & -0.35580000 & -0.45880000 \\
\hline $\mathrm{C}$ & -4.42670000 & -1.57580000 & 1.13200000 \\
\hline $\mathrm{H}$ & -3.42610000 & -1.56890000 & 1.57420000 \\
\hline C & -4.58640000 & -2.99840000 & 0.5415000 \\
\hline $\mathrm{C}$ & -5.44040000 & -1.05880000 & 2.16340000 \\
\hline $\mathrm{H}$ & -5.18910000 & -0.00970000 & 2.35420000 \\
\hline $\mathrm{H}$ & -6.44190000 & -1.06570000 & 1.71440000 \\
\hline $\mathrm{C}$ & -5.47340000 & -1.85690000 & 3.49090000 \\
\hline $\mathrm{H}$ & -4.82190000 & -2.74080000 & 3.41600000 \\
\hline $\mathrm{H}$ & -5.09360000 & -1.25470000 & 4.32140000 \\
\hline $\mathrm{C}$ & -6.89880000 & -2.36680000 & 3.82860000 \\
\hline O & -7.27440000 & -2.35580000 & 5.02210000 \\
\hline O & -7.59590000 & -2.74560000 & 2.81740000 \\
\hline O & -3.87420000 & -3.32490000 & -0.43300000 \\
\hline $\mathrm{N}$ & -5.44480000 & -3.84830000 & 1.12750000 \\
\hline $\mathrm{H}$ & -6.07560000 & -3.61440000 & 1.95410000 \\
\hline C & -5.55350000 & -5.18720000 & 0.59290000 \\
\hline $\mathrm{H}$ & -5.92750000 & -5.85030000 & 1.37730000 \\
\hline $\mathrm{H}$ & -4.57240000 & -5.53530000 & 0.26050000 \\
\hline C & -1.71080000 & 4.48880000 & 1.21480000 \\
\hline $\mathrm{H}$ & -1.68210000 & 3.50160000 & 1.68550000 \\
\hline C & -3.13750000 & 4.62050000 & 0.6270000 \\
\hline C & -1.23660000 & 5.55790000 & 2.2088000 \\
\hline $\mathrm{H}$ & -0.15730000 & 5.43250000 & 2.3475000 \\
\hline $\mathrm{H}$ & -1.39220000 & 6.54670000 & 1.75870000 \\
\hline C & -1.95330000 & 5.50000000 & 3.58100000 \\
\hline $\mathrm{H}$ & -2.69360000 & 4.68560000 & 3.58850000 \\
\hline $\mathrm{H}$ & -1.24380000 & 5.28310000 & 4.38570000 \\
\hline C & -2.74850000 & 6.79340000 & 3.88640000 \\
\hline 0 & -3.39250000 & 7.27350000 & 2.8838000 \\
\hline O & -2.78580000 & 7.21390000 & 5.0637000 \\
\hline 0 & -3.51400000 & 3.80170000 & -0.2366000 \\
\hline $\mathrm{N}$ & -3.94650000 & 5.57770000 & 1.1075000 \\
\hline $\mathrm{H}$ & -3.64890000 & 6.33940000 & 1.7843000 \\
\hline C & -5.31010000 & 5.61980000 & 0.63360000 \\
\hline $\mathrm{H}$ & -5.92780000 & 6.12690000 & 1.37900000 \\
\hline $\mathrm{H}$ & -5.67820000 & 4.60000000 & 0.49400000 \\
\hline C & 4.48490000 & 1.70670000 & 1.2671000 \\
\hline $\mathrm{H}$ & 3.48170000 & 1.86190000 & 1.67530000 \\
\hline $\mathrm{C}$ & 4.85770000 & 3.03140000 & 0.5567000 \\
\hline
\end{tabular}




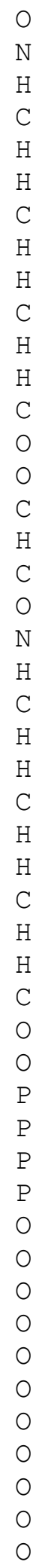

\begin{tabular}{|c|c|c|}
\hline 4.30200000 & 3.31650000 & -0.52290000 \\
\hline 5.79510000 & 3.82010000 & 1.10170000 \\
\hline 6.38640000 & 3.56450000 & 1.94950000 \\
\hline 6.06150000 & 5.08150000 & 0.44810000 \\
\hline 6.49000000 & 5.77810000 & 1.17390000 \\
\hline 5.12450000 & 5.49380000 & 0.06410000 \\
\hline 5.42490000 & 1.19860000 & 2.36790000 \\
\hline 5.15410000 & 0.15870000 & 2.58010000 \\
\hline 6.45170000 & 1.19290000 & 1.97970000 \\
\hline 5.36320000 & 2.02990000 & 3.67510000 \\
\hline 4.95590000 & 1.43070000 & 4.49500000 \\
\hline 4.68490000 & 2.88700000 & 3.54730000 \\
\hline $6.73 \varepsilon$ & 2.61810000 & 4.07330000 \\
\hline 7.35140000 & 3.21190000 & 3.11 \\
\hline 7.00370000 & 2.73450000 & 5.29000000 \\
\hline 1.51940000 & -4.38040000 & 1.20150000 \\
\hline 1.51180000 & -3.40380000 & 1.69760000 \\
\hline 2.92970000 & -4.54630000 & 0.60010000 \\
\hline 3.28870000 & -3.78990000 & -0.32800000 \\
\hline 3.71570000 & -5.49030000 & 1.13740000 \\
\hline 3.36870000 & -6.19470000 & 1.86050000 \\
\hline 5.08520000 & -5.59650000 & 0.67600 \\
\hline 5.486 & -4.5954 & $0.497 \xi$ \\
\hline 5.68040000 & -6.0889 & 1.44930000 \\
\hline 1.02780000 & -5.45500000 & 2.18140000 \\
\hline-0.04820000 & -5.29070000 & 2.30500000 \\
\hline 1.15540000 & -6.44590000 & 1.72620000 \\
\hline 1.70990000 & -5.41340000 & 3.57480000 \\
\hline 2.51400000 & -4.66340000 & 3.5863000 \\
\hline 0.98810000 & -5.10050000 & 4.3359000 \\
\hline 2.35620000 & -6.75570000 & 4.0005000 \\
\hline 2.33 & -7.05100000 & 5.215700 \\
\hline 3.08560000 & -7.32020000 & 3.105100 \\
\hline 7.22290000 & 4.97920000 & -0.99730000 \\
\hline-5.55060000 & 6.50520000 & -0.9790000 \\
\hline-6.69790000 & -5.33920000 & -0.8622000 \\
\hline 5.32670000 & -6.54920000 & -0.9033000 \\
\hline 6.07230000 & -5.79930000 & -1.9840000 \\
\hline-6.09160000 & -5.98940000 & -2.0842000 \\
\hline-6.27010000 & 5.69220000 & -2.028300 \\
\hline 6.68250000 & 5.61020000 & -2.2585000 \\
\hline 8.65290000 & 5.59640000 & -0.51630000 \\
\hline 7.58070000 & 3.40610000 & -1.01850000 \\
\hline-4.05000000 & 7.01910000 & -1.2744000 \\
\hline-6.27900000 & 7.92610000 & -0.6392000 \\
\hline 6.03670000 & -7.96270000 & -0.5031000 \\
\hline 3.8241000 & -7.06460000 & -1.1945000 \\
\hline-8.0 & -6.08480000 & -0.3327000 \\
\hline
\end{tabular}




\begin{tabular}{lrrr}
$\mathrm{O}$ & -7.23730000 & -3.82100000 & -0.95850000 \\
$\mathrm{C}$ & 3.50770000 & -7.73150000 & -2.43610000 \\
$\mathrm{H}$ & 3.73200000 & -7.09020000 & -3.29530000 \\
$\mathrm{H}$ & 4.07040000 & -8.66410000 & -2.50890000 \\
$\mathrm{H}$ & 2.43650000 & -7.93270000 & -2.40310000 \\
$\mathrm{C}$ & 7.44910000 & -8.14790000 & -0.70890000 \\
$\mathrm{H}$ & 7.79500000 & -7.59810000 & -1.58810000 \\
$\mathrm{H}$ & 8.00830000 & -7.82820000 & 0.17710000 \\
$\mathrm{H}$ & 7.58430000 & -9.21990000 & -0.85770000 \\
$\mathrm{C}$ & -7.96060000 & -3.38850000 & -2.13210000 \\
$\mathrm{H}$ & -7.35660000 & -3.51900000 & -3.03690000 \\
$\mathrm{H}$ & -8.89280000 & -3.95080000 & -2.21780000 \\
$\mathrm{H}$ & -8.16620000 & -2.32820000 & -1.98350000 \\
$\mathrm{C}$ & -8.28460000 & -7.47690000 & -0.62190000 \\
$\mathrm{H}$ & -7.91620000 & -7.74430000 & -1.61560000 \\
$\mathrm{H}$ & -7.81860000 & -8.11830000 & 0.13350000 \\
$\mathrm{H}$ & -9.36740000 & -7.59960000 & -0.57850000 \\
$\mathrm{C}$ & 8.47430000 & 2.85130000 & -2.00910000 \\
$\mathrm{H}$ & 8.11200000 & 3.05770000 & -3.02170000 \\
$\mathrm{H}$ & 9.47780000 & 3.26190000 & -1.88090000 \\
$\mathrm{H}$ & 8.47820000 & 1.77450000 & -1.83740000 \\
$\mathrm{C}$ & 8.97540000 & 6.97190000 & -0.79000000 \\
$\mathrm{H}$ & 8.52000000 & 7.30750000 & -1.72560000 \\
$\mathrm{H}$ & 8.64680000 & 7.61050000 & 0.03770000 \\
$\mathrm{H}$ & 10.06270000 & 7.00810000 & -0.86630000 \\
$\mathrm{C}$ & -3.72410000 & 7.64250000 & -2.53620000 \\
$\mathrm{H}$ & -3.94170000 & 6.97160000 & -3.37420000 \\
$\mathrm{H}$ & -4.28600000 & 8.57220000 & -2.64610000 \\
$\mathrm{H}$ & -2.65330000 & 7.84500000 & -2.50150000 \\
$\mathrm{C}$ & -7.70250000 & 8.08230000 & -0.80070000 \\
$\mathrm{H}$ & -8.23360000 & 7.68230000 & 0.07050000 \\
$\mathrm{H}$ & -7.88580000 & 9.15550000 & -0.87820000 \\
$\mathrm{H}$ & -8.04290000 & 7.57520000 & -1.70760000 \\
\hline & &
\end{tabular}

EuL1, TPSSh/LCRECP/6-31G(d), aqueous solution

\begin{tabular}{lrrr}
\hline $\mathrm{N}$ & -1.82575900 & 1.10915000 & -1.98977600 \\
$\mathrm{C}$ & -2.14192000 & 0.15852300 & -3.10589400 \\
$\mathrm{C}$ & -2.27695600 & -1.29216200 & -2.66403500 \\
$\mathrm{~N}$ & -1.02690300 & -1.81050600 & -2.03006700 \\
$\mathrm{C}$ & -0.01498300 & -2.13082400 & -3.08700700 \\
$\mathrm{C}$ & 1.40794700 & -2.26865900 & -2.55871700 \\
$\mathrm{~N}$ & 1.89686000 & -1.01238200 & -1.91261300 \\
$\mathrm{C}$ & 2.27847000 & -0.02381800 & -2.96517400 \\
$\mathrm{C}$ & 2.38863300 & 1.41383300 & -2.46503800 \\
$\mathrm{~N}$ & 1.10476800 & 1.91258400 & -1.88238500 \\
$\mathrm{C}$ & 0.15763300 & 2.28129500 & -2.98125100 \\
$\mathrm{C}$ & -1.29994300 & 2.39129900 & -2.55110800
\end{tabular}




\begin{tabular}{|c|c|c|c|}
\hline $\mathrm{C}$ & -3.04010200 & 1.38903200 & -1.18241400 \\
\hline $\mathrm{C}$ & -3.26903900 & 0.30244400 & -0.14517000 \\
\hline 0 & -2.28237200 & -0.27935400 & 0.38353900 \\
\hline $\mathrm{C}$ & -1.35273900 & -3.02415200 & -1.24117800 \\
\hline C & -0.32631200 & -3.25163000 & -0.14783600 \\
\hline 0 & 0.21768800 & -2.26246700 & 0.41589100 \\
\hline C & 3.06119200 & -1.33435400 & -1.05119400 \\
\hline $\mathrm{C}$ & 3.24591000 & -0.28734700 & 0.03066500 \\
\hline 0 & 2.23188900 & 0.26367900 & 0.54623800 \\
\hline $\mathrm{C}$ & 1.38376100 & 3.09041900 & -1.02498100 \\
\hline C & 0.27562800 & 3.28432500 & -0.00596300 \\
\hline 0 & -0.32521300 & 2.27999000 & 0.46382700 \\
\hline $\mathrm{H}$ & -1.35231000 & 0.23251200 & -3.85946600 \\
\hline $\mathrm{H}$ & -3.07634800 & 0.46143900 & -3.59984000 \\
\hline $\mathrm{H}$ & -3.09261600 & -1.41764300 & -1.94738100 \\
\hline $\mathrm{H}$ & -2.53510000 & -1.89423700 & -3.54726800 \\
\hline $\mathrm{H}$ & -0.04481500 & -1.34515900 & -3.84782900 \\
\hline $\mathrm{H}$ & -0.29063300 & -3.06739800 & -3.59245300 \\
\hline $\mathrm{H}$ & 1.48794700 & -3.07498600 & -1.82550300 \\
\hline $\mathrm{H}$ & 2.05975200 & -2.54019100 & -3.40134400 \\
\hline $\mathrm{H}$ & 1.53099400 & -0.07682100 & -3.75867300 \\
\hline $\mathrm{H}$ & 3.23892100 & -0.31272200 & -3.41530400 \\
\hline $\mathrm{H}$ & 3.16465400 & 1.51273000 & -1.70204300 \\
\hline $\mathrm{H}$ & 2.69387300 & 2.04851300 & -3.30946900 \\
\hline $\mathrm{H}$ & 0.24411700 & 1.53686600 & -3.76971500 \\
\hline $\mathrm{H}$ & 0.46290100 & 3.24055300 & -3.42344900 \\
\hline $\mathrm{H}$ & -1.43640800 & 3.16913700 & -1.79638000 \\
\hline $\mathrm{H}$ & -1.89164600 & 2.69310100 & -3.42748600 \\
\hline $\mathrm{H}$ & -2.87579600 & 2.32595600 & -0.63439400 \\
\hline $\mathrm{H}$ & -3.92360500 & 1.52593000 & -1.82092700 \\
\hline $\mathrm{H}$ & -2.31906300 & -2.86524100 & -0.74471700 \\
\hline $\mathrm{H}$ & -1.45071700 & -3.90615000 & -1.8888050 \\
\hline $\mathrm{H}$ & 2.86038300 & -2.28757900 & -0.5442930 \\
\hline $\mathrm{H}$ & 3.97648200 & -1.45851800 & -1.6460710 \\
\hline $\mathrm{H}$ & 2.30971300 & 2.89896000 & -0.4672200 \\
\hline $\mathrm{H}$ & 1.53885500 & 3.99670900 & -1.62688500 \\
\hline 0 & 0.03556000 & -0.07345600 & 2.17488900 \\
\hline $\mathrm{H}$ & -0.18605800 & -0.97653500 & 2.47089500 \\
\hline $\mathrm{H}$ & 0.97798100 & 0.04189700 & 2.40635500 \\
\hline $\mathrm{Eu}$ & -0.01423700 & 0.02244400 & -0.3410850 \\
\hline $\mathrm{N}$ & 0.01945800 & 4.52640700 & 0.3997850 \\
\hline $\mathrm{N}$ & 4.48776700 & -0.03891400 & 0.4371190 \\
\hline $\mathrm{N}$ & -0.08131700 & -4.50545200 & 0.2220160 \\
\hline $\mathrm{N}$ & -4.52037700 & 0.05020700 & 0.2329410 \\
\hline $\mathrm{H}$ & 0.51167300 & 5.28898100 & -0.0548410 \\
\hline $\mathrm{H}$ & 5.24835000 & -0.48931800 & -0.0625240 \\
\hline $\mathrm{H}$ & -0.51804100 & -5.25481500 & -0.3059610 \\
\hline $\mathrm{H}$ & -5.27709800 & 0.51802500 & -0.2559450 \\
\hline
\end{tabular}




\begin{tabular}{|c|c|c|c|}
\hline $\mathrm{C}$ & -4.87535700 & -0.93063000 & 1.26662900 \\
\hline $\mathrm{H}$ & -3.94578700 & -1.12095500 & 1.81262700 \\
\hline C & -5.23092200 & -2.25659300 & 0.55452500 \\
\hline C & -5.92348200 & -0.34488900 & 2.22705800 \\
\hline $\mathrm{H}$ & -5.62728600 & 0.69079700 & 2.42406800 \\
\hline $\mathrm{H}$ & -6.89898200 & -0.31379200 & 1.72731100 \\
\hline C & -6.04470800 & -1.11117000 & 3.56940100 \\
\hline $\mathrm{H}$ & -5.37999600 & -1.98801400 & 3.56231600 \\
\hline $\mathrm{H}$ & -5.71447600 & -0.48126800 & 4.40009600 \\
\hline C & -7.47334900 & -1.63328200 & 3.86684100 \\
\hline 0 & -7.91705800 & -1.51010500 & 5.03001800 \\
\hline O & -8.08601800 & -2.16750800 & 2.8737390 \\
\hline O & -4.50224600 & -2.68156300 & -0.36269200 \\
\hline $\mathrm{N}$ & -6.30672400 & -2.92326500 & 1.00031000 \\
\hline $\mathrm{H}$ & -6.98365600 & -2.53236800 & 1.71890800 \\
\hline C & -6.62924600 & -4.21291100 & 0.42738600 \\
\hline $\mathrm{H}$ & -7.25593800 & -4.75661100 & 1.13905300 \\
\hline $\mathrm{H}$ & -5.71335200 & -4.78189800 & 0.2453220 \\
\hline C & -0.99857900 & 4.87863300 & 1.3977780 \\
\hline $\mathrm{H}$ & -1.22235400 & 3.95001000 & 1.93187500 \\
\hline C & -2.28927800 & 5.25123000 & 0.62898900 \\
\hline C & -0.43323500 & 5.91892400 & 2.37943700 \\
\hline $\mathrm{H}$ & 0.57567000 & 5.58586900 & 2.64479800 \\
\hline $\mathrm{H}$ & -0.33282600 & 6.88301700 & 1.86666300 \\
\hline C & -1.27453600 & 6.10494400 & 3.66749400 \\
\hline $\mathrm{H}$ & -2.17811600 & 5.47878700 & 3.61821500 \\
\hline $\mathrm{H}$ & -0.71390900 & 5.77021100 & 4.5446220 \\
\hline $\mathrm{C}$ & -1.74947100 & 7.56347100 & 3.8952730 \\
\hline 0 & -2.17378700 & 8.17883800 & 2.8525700 \\
\hline 0 & -1.72365100 & 8.01698000 & 5.06113400 \\
\hline O & -2.70706600 & 4.49966200 & -0.27306300 \\
\hline $\mathrm{N}$ & -2.93295400 & 6.36972600 & 0.9962480 \\
\hline $\mathrm{H}$ & -2.55435300 & 7.05899600 & 1.70633200 \\
\hline C & -4.17366900 & 6.71904300 & 0.3375260 \\
\hline $\mathrm{H}$ & -4.69960800 & 7.44297500 & 0.9649460 \\
\hline $\mathrm{H}$ & -4.79764300 & 5.82924400 & 0.2138700 \\
\hline C & 4.85199700 & 0.92185400 & 1.4880270 \\
\hline $\mathrm{H}$ & 3.92685000 & 1.12819400 & 2.0340980 \\
\hline C & 5.23428500 & 2.24361900 & 0.77963400 \\
\hline O & 4.44696800 & 2.74539100 & -0.0459810 \\
\hline $\mathrm{N}$ & 6.40906700 & 2.80739300 & 1.0975380 \\
\hline $\mathrm{H}$ & 7.10933900 & 2.37498800 & 1.7686210 \\
\hline C & 6.76225000 & 4.07362500 & 0.4921580 \\
\hline $\mathrm{H}$ & 7.52528200 & 4.55093400 & 1.1127040 \\
\hline $\mathrm{H}$ & 5.88323100 & 4.72224000 & 0.4423690 \\
\hline $\mathrm{C}$ & 5.88776800 & 0.30260000 & 2.4383700 \\
\hline $\mathrm{H}$ & 5.53127700 & -0.70305400 & 2.6848460 \\
\hline $\mathrm{H}$ & 6.84226200 & 0.18892000 & 1.9105950 \\
\hline
\end{tabular}




\begin{tabular}{|c|c|c|c|}
\hline $\mathrm{C}$ & 6.10786700 & 1.11157400 & 3.74199700 \\
\hline $\mathrm{H}$ & 5.76855200 & 0.53976800 & 4.61007200 \\
\hline $\mathrm{H}$ & 5.50280000 & 2.03060300 & 3.72110300 \\
\hline C & 7.57869200 & 1.54773800 & 3.96512100 \\
\hline O & 8.19738900 & 1.96435100 & 2.92069700 \\
\hline 0 & 8.04837300 & 1.47231900 & 5.12198900 \\
\hline C & 0.85860300 & -4.88372500 & 1.28405900 \\
\hline $\mathrm{H}$ & 1.01223100 & -3.97535900 & 1.87525300 \\
\hline C & 2.21629800 & -5.18724100 & 0.60891000 \\
\hline O & 2.66984400 & -4.39984400 & -0.24424700 \\
\hline N & 2.86968300 & -6.29178900 & 0.9997550 \\
\hline $\mathrm{H}$ & 2.45856500 & -7.01746000 & 1.65776200 \\
\hline C & 4.17565100 & -6.57100100 & 0.44075800 \\
\hline $\mathrm{H}$ & 4.77093100 & -5.65445600 & 0.39867600 \\
\hline $\mathrm{H}$ & 4.67715800 & -7.29552100 & 1.08724400 \\
\hline C & 0.24960100 & -5.98176600 & 2.1709810 \\
\hline $\mathrm{H}$ & -0.79121500 & -5.69677100 & 2.3571600 \\
\hline $\mathrm{H}$ & 0.23244400 & -6.92878400 & 1.61836000 \\
\hline C & 0.98309100 & -6.18078800 & 3.52170100 \\
\hline $\mathrm{H}$ & 1.86153600 & -5.52009700 & 3.5736590 \\
\hline $\mathrm{H}$ & 0.33402900 & -5.89714500 & 4.35466000 \\
\hline C & 1.49198000 & -7.62762300 & 3.74561500 \\
\hline 0 & 1.34708900 & -8.13675800 & 4.8790790 \\
\hline O & 2.05315700 & -8.18034100 & 2.7321760 \\
\hline $\mathrm{P}$ & 7.42479400 & 3.97015300 & -1.2365400 \\
\hline $\mathrm{P}$ & -3.99103700 & 7.45984700 & -1.3531660 \\
\hline $\mathrm{P}$ & -7.53300700 & -4.15374800 & -1.19011400 \\
\hline $\mathrm{P}$ & 4.16450800 & -7.25833900 & -1.28202200 \\
\hline 0 & 4.85648400 & -6.42840700 & -2.3154220 \\
\hline O & -6.86607600 & -4.86059800 & -2.3264500 \\
\hline O & -4.67401800 & 6.71833800 & -2.4561910 \\
\hline O & 6.75525000 & 4.88014600 & -2.2157360 \\
\hline O & 9.03262700 & 4.19018200 & -1.1334160 \\
\hline O & 7.37159000 & 2.38797800 & -1.5299080 \\
\hline 0 & -2.39564200 & 7.65193300 & -1.4622530 \\
\hline O & -4.46338400 & 9.01229900 & -1.2252030 \\
\hline O & 4.74977500 & -8.77195600 & -1.16692900 \\
\hline O & 2.59758900 & -7.56265300 & -1.5015030 \\
\hline O & -9.04104400 & -4.68457600 & -0.8868360 \\
\hline O & -7.80572500 & -2.57551600 & -1.3566910 \\
\hline C & 2.18651300 & -8.19128000 & -2.741878 \\
\hline $\mathrm{H}$ & 2.48911300 & -7.57798600 & -3.594238 \\
\hline $\mathrm{H}$ & 2.62580600 & -9.18897700 & $-2.817737 c$ \\
\hline $\mathrm{H}$ & 1.10019800 & -8.26030800 & -2.6946010 \\
\hline C & 6.13474400 & -9.04079100 & -1.4848570 \\
\hline $\mathrm{H}$ & 6.44884800 & -8.45045100 & -2.3481990 \\
\hline $\mathrm{H}$ & 6.76729400 & -8.81220300 & -0.6220810 \\
\hline $\mathrm{H}$ & 6.19122500 & -10.10549500 & -1.7132340 \\
\hline
\end{tabular}




\begin{tabular}{lrrr}
$\mathrm{C}$ & -8.55129300 & -2.12572300 & -2.51638200 \\
$\mathrm{H}$ & -8.05508900 & -2.45140100 & -3.43383100 \\
$\mathrm{H}$ & -9.57132500 & -2.51596800 & -2.47785100 \\
$\mathrm{H}$ & -8.56187400 & -1.03780600 & -2.45894700 \\
$\mathrm{C}$ & -9.38040600 & -6.06971300 & -1.12845300 \\
$\mathrm{H}$ & -8.94101000 & -6.41162800 & -2.06786200 \\
$\mathrm{H}$ & -9.02880400 & -6.69225800 & -0.30027300 \\
$\mathrm{H}$ & -10.46863700 & -6.10794400 & -1.18283400 \\
$\mathrm{C}$ & 7.91521200 & 1.89506900 & -2.78049700 \\
$\mathrm{H}$ & 7.40227600 & 2.36204700 & -3.62518400 \\
$\mathrm{H}$ & 8.98732800 & 2.09975000 & -2.82745100 \\
$\mathrm{H}$ & 7.73476400 & 0.82059200 & -2.78003200 \\
$\mathrm{C}$ & 9.59807400 & 5.50615700 & -1.33373600 \\
$\mathrm{H}$ & 9.08073700 & 6.02470400 & -2.14384600 \\
$\mathrm{H}$ & 9.52773300 & 6.08663700 & -0.40908800 \\
$\mathrm{H}$ & 10.64532300 & 5.34885900 & -1.59318200 \\
$\mathrm{C}$ & -1.85868400 & 8.29703400 & -2.64478400 \\
$\mathrm{H}$ & -2.17255100 & 7.76028600 & -3.54358800 \\
$\mathrm{H}$ & -2.19425100 & 9.33601500 & -2.68717000 \\
$\mathrm{H}$ & -0.77498600 & 8.25417400 & -2.54085400 \\
$\mathrm{C}$ & -5.81720000 & 9.38590700 & -1.57109600 \\
$\mathrm{H}$ & -6.49237900 & 9.15598500 & -0.74143800 \\
$\mathrm{H}$ & -5.79884000 & 10.46218200 & -1.74473900 \\
$\mathrm{H}$ & -6.13889300 & 8.86144800 & -2.47337700 \\
\hline & & &
\end{tabular}

\section{REFERENCES}

(1) Kálmán, F. K.; Woods, M.; Caravan, P.; Jurek, P.; Spiller, M.; Tircsó, G.; Király, R.; Brücher, E.; Sherry, A. D. Inorg. Chem. 2007, 46 , 5260-5270.

(2) Ali, M. M.; Woods, M.; Caravan, P.; Opina, A. C. L.; Spiller, M.; Fettinger, J. C.; Sherry, A. D. Chem. Weinh. Bergstr. Ger. 2008, 14, 7250-7258.

\section{FULL REFERENCE 23 (MAIN MANUSCRIPT):}

(1) Kálmán Frisch, M. J.; Trucks, G. W.; Schlegel, H. B.; Scuseria, G. E.; Robb, M. A.; Cheeseman, J. R.; Scalmani, G.; Barone, V.; Mennucci, B.; Petersson, G. A.; Nakatsuji, H.; Caricato, M.; Li, X.; Hratchian, H. P.; Izmaylov, A. F.; Bloino, J.; Zheng, G.; Sonnenberg, J. L.; Hada, M.; Ehara, M.; Toyota, K.; Fukuda, R.; Hasegawa, J.; Ishida, M.; Nakajima, T.; Honda, Y.; Kitao, O.; Nakai, H.; Vreven, T.; Montgomery, J. A., Jr.; Peralta, J. E.; Ogliaro, F.; Bearpark, M.; Heyd, J. J.; Brothers, E.; Kudin, K. N.; Staroverov, V. N.; Kobayashi, R.; Normand, J.; Raghavachari, K.; Rendell, A.; Burant, J. C.; Iyengar, S. S.; Tomasi, J.; Cossi, M.; Rega, N.; Millam, J. M.; Klene, M.; Knox, J. 
E.; Cross, J. B.; Bakken, V.; Adamo, C.; Jaramillo, J.; Gomperts, R.; Stratmann, R. E.; Yazyev, O.; Austin, A. J.; Cammi, R.; Pomelli, C.; Ochterski, J. W.; Martin, R. L.; Morokuma, K.; Zakrzewski, V. G.; Voth, G. A.; Salvador, P.; Dannenberg, J. J.; Dapprich, S.; Daniels, A. D.; Farkas, Ö.; Foresman, J. B.; Ortiz, J. V.; Cioslowski, J.; Fox, D. J. Gaussian o9 Revision D.o1. 